\title{
Transitions between streamline topologies of structurally stable Hamiltonian flows in multiply connected domains
}

\author{
AUTHOR(S): \\ Sakajo, Takashi; Yokoyama, Tomoo
}

\section{CITATION:}

Sakajo, Takashi ...[et al]. Transitions between streamline to pologies of structurally stable Hamiltonian flows in multiply connected domains. Physica D: Nonlinear Phenomena 2015, 307: 22-41

ISSUE DATE:

2015-07-01

URL:

http://hdl.handle.net/2433/201596

\section{RIGHT:}

(c) 2015. This manuscript version is made available under the CC-BY-NC-ND 4.0 license

http://creativecommons.org/licenses/by-nc-nd/4.0/; The full-text file will be made open to the public on $1 \mathrm{July} 2017$ in accordance with publisher's 'Terms and Conditions for Self-Archiving'.; この論文は出版社版でありません。引用の際に は出版社版をご確認ご利用ください。; This is not the published version. Please cite only the published version. 


\title{
Transitions between streamline topologies of structurally stable Hamiltonian flows in multiply connected domains
}

\author{
Takashi Sakajo*and Tomoo Yokoyama ${ }^{\dagger}$
}

August 14, 2015

\begin{abstract}
We consider Hamiltonian vector fields with a dipole singularity satisfying the slip boundary condition in two-dimensional multiply connected domains. One example of such Hamiltonian vector fields is an incompressible and inviscid flow in exterior multiply connected domains with a uniform flow, whose Hamiltonian is called the stream function. Here, we are concerned with topological structures of the level sets of the Hamiltonian, which we call streamlines by analogy from incompressible fluid flows. Classification of structurally stable streamline patterns has been considered in [15], where a procedure to assign a unique sequence of words, called the maximal word, to these patterns is proposed. Thanks to this procedure, we can identify every streamline pattern with its representing sequence of words up to topological equivalence. In the present paper, based on the theory of word representations, we propose a combinatorial method to provide a list of possible transient structurally unstable streamline patterns between two different structurally stable patterns by simply comparing their maximal word representations without specifying any Hamiltonian. Although this method can not deal with topological streamline changes induced by bifurcations, it reveals the existence of many non-trivial global transitions in a generic sense. We also demonstrate how the present theory is applied to fluid flow problems with vortex structures.
\end{abstract}

Keywords: Hamiltonian flows; streamline topology; transition; structural stability; vortex dynamics

MSC: 76B47; 37E35; 37G20; 34D30

\section{Introduction}

Flow problems in two-dimensional multiply connected domains in the presence of a uniform flow are of significance from an applications point of view, since they are often regarded as mathematical models of biofluids and environmental flows such as the schooling of fish in rivers and coastal current flows in the presence of many islands. Suppose here that the flow is incompressible. Then the instantaneous velocity field $(u(t, x, y), v(t, x, y))$ at a location $(x, y)$ and at time $t$ is given by $u=\partial_{y} \psi$ and $v=-\partial_{x} \psi$ for a stream function $\psi(t, x, y)$, which gives rise to a Hamiltonian vector field with $\psi$ being the Hamiltonian. In this paper, we are concerned with topological structures

\footnotetext{
${ }^{*}$ Department of mathematics, Kyoto University, Sakyo-ku, Kyoto, 606-8502 Japan \& JST CREST, Kyoto E-mail: sakajo@math.kyoto-u.ac.jp

${ }^{\dagger}$ Department of mathematics, Kyoto University of Education, Fushimi-ku, Kyoto 616-8522 Japan \& JST CREST, Kyoto E-mail: tomoo@kyokyo-u.ac.jp
} 
of level sets of the stream function, called streamlines, and their transitions. Streamline topology plays a significant role in characterizing incompressible flows and has been considered in many fluid problems. For instance, Brøns et al.[7] described the bifurcations of streamline topologies of a flow around a circular cylinder at moderate Reynolds numbers. Brøns and Hartnack[8] also investigated the bifurcations of streamline patterns of the steady Navier-Stokes, or Stokes flows, near some degenerate critical points away from boundaries. More examples are found in $[3,4,5,6,10]$. These studies are based on the local bifurcation analysis of the Hamiltonian associated with the fluid problems.

In the meantime, there is a combinatorial approach to investigate streamline topologies. For example, Aref and Brøns[1] have considered a classification of streamline topologies generated by potential flows with point-vortex singularities, called vortex flows, in the 2D unbounded plane. This study has been extended to the Hamiltonian vector fields, which are a generalization of the vortex flows, in 2D multiply connected domains[13, 15], in which the classification of structurally stable streamline topologies that are unchanged under small perturbations are considered. In these studies, the Hamiltonian vector fields are assumed to satisfy the slip boundary condition. The classification theory also provides a procedure to assign a unique sequence of words to every streamline topology, which allows us to identify each structurally stable streamline pattern with this sequence of words up to topological equivalence. In addition, it is possible to describe an evolution of streamline topologies as a change of words by assigning the unique sequence of word to an instantaneous streamline patterns at each time of the evolution.

When a transition of streamline topologies is represented by a change of the words, it is natural to ask whether a transient streamline pattern that lies between these patterns can be determined from their word representations. This is not an easy task, since the transient streamline pattern is structurally unstable and it may thus contain many singular streamline structures[9]. In the present study, we propose a combinatorial method providing a list of possible transitions between two structurally stable streamline topologies from their word representations. It is also able to show which transitions are impossible by just comparing the word representations. The construction of this paper is as follows. In the next section, we review the theory of word representations for structurally stable Hamiltonian vector fields in multiply connected domains developed in [13, 15]. We then make some preparations for describing transitions between structurally stable Hamiltonian vector fields. In particular, we introduce two special sets of structurally unstable Hamiltonian vector fields, called $h$-unstable and $p$-unstable vector fields. We use these unstable vector fields to describe transient streamline patterns, since it is mathematically shown in $\S 3$ that they are open dense in the set of structurally unstable vector fields under certain assumptions. In $\S \S 4$ and 5 , we introduce some operations that generate $h$-unstable and $p$-unstable streamline patterns from structurally stable patterns and observe how small perturbations of these unstable patterns give rise to changes of the structurally stable patterns and their word representations. Let us remark that, in contrast to the bifurcation analysis in the preceding studies, our mathematical approach is combinatorial and no mathematical analysis of the Hamiltonian is required. In $\S 6$, we explain how the present theory is applied to describing transitions of streamline topologies of fluid flows from their word representations. In $\S 7$, we provide some applications to incompressible fluid flows to figure out some features of the combinatorial method and differences from the preceding bifurcation analysis[7, 10]. In the final section, we summarize the global transition analysis given in this paper and we then discuss about its significance. 


\section{Word representation for structurally stable Hamiltonian vector fields}

The global transition analysis given in this paper is based on the theory of word representations for streamline topologies of structurally stable Hamiltonian vector fields satisfying the slip boundary condition in two-dimensional multiply connected domains with a dipole singularity[15], which is reviewed as follows. In order to characterize multiply connected domains topologically, we use the term genus element instead of genus usually used in mathematical studies. A genus element represents not only a physical obstacle and a singular point where the value of the Hamiltonian diverges, but also an elliptic fixed point of the vector field. This is an unconventional but necessary term, since elliptic fixed points can change to saddle fixed points owing to the bifurcation and then they are not regarded as genus. However, in the present paper, assuming that no bifurcation generating or removing elliptic fixed points occurs, we restrict our attention to the global transitions between structurally stable streamline topologies with the same number of genus elements. Therefore, it is unnecessary to distinguish physical obstacles, singular points and elliptic fixed points, and thus all genus elements are schematically represented as circular holes in the following illustrations. This means that the number of genus elements does not always coincide with that of physical obstacles in the domain. In other words, any circular obstacle without saddle points at its boundary can be replaced by a singular point or an elliptic fixed point. Let us remark that the bifurcation of fixed points is an interesting phenomenon mathematically as well as physically even if it is prohibited in this paper. We will show some examples in $\S 7$ to see the difference between the changes of topological streamline patterns handled by the present theory and those by the bifurcation analysis, and we then discuss more about it in the last section.

Since the uniform flow exists in unbounded domains, we need to consider an exterior domain with $M$ genus elements in the complex $z$-plane, which is denoted by $\mathcal{D}_{z}(M)$. Since all genus elements are represented as circular holes in this paper, the domain has $M+1$ circular boundaries. In what follows for a technical reason, we consider the topological streamline structures of Hamiltonian vector fields in a multiply connected bounded domain $\mathcal{D}_{\zeta}(M)$ with the same number of genus elements in the complex $\zeta$-plane by constructing the conformal mapping from $\mathcal{D}_{z}(M)$ to $\mathcal{D}_{\zeta}(M)$. This causes no serious problem, since the streamline topologies are invariant under the action of the conformal mapping. Then the uniform flow is characterized in terms of a Hamiltonian vector field in the bounded domain as follows. Since the uniform flow is irrotational, it is represented by a complex potential $W_{U}(z)$ that behaves like $W_{U}(z) \sim U \mathrm{e}^{-i \phi} z$ as $z \rightarrow \infty$ for the flux $U$ and the angle of inclination to the real axis $\phi$ in the exterior domain $\mathcal{D}_{z}(M)$. For a conformal mapping $z=f(\zeta)$ from the bounded multiply connected $\mathcal{D}_{\zeta}(M)$ to $\mathcal{D}_{z}(M)$ with $f(0)=\infty$, the asymptotic behavior of the conformal mapping in the neighborhood of the origin becomes $z=f(\zeta) \sim \frac{a}{\zeta}$ for some constant $a$. Hence, the complex potential of the uniform flow is given by $W_{U}(\zeta) \sim U \mathrm{e}^{-i \phi} \frac{a}{\zeta}$ as $\zeta \rightarrow 0$. Here we may set $U=1, \phi=0$ and $a=1$ without loss of generality, since we are interested in the topological streamline structure of the uniform flow in the neighborhood of the origin. We call the singular point at the origin of $\mathcal{D}_{\zeta}(M)$ the 1-source-sink point whose definition is given as follows[15].

Definition 2.1. A point $p \in \mathcal{D}_{\zeta}(M)$ is said to be a 1-source-sink point, if $\left.V\right|_{\mathcal{D}_{\zeta}(M) \backslash\{p\}}$ is a vector field on $\mathcal{D}_{\zeta}(M) \backslash\{p\}$ generated by a stream function $\psi$, for which there is a pair of a neighborhood $U$ of $p$ and a homeomorphism $h$ from $U$ to the unit disk $D$ with $h(p)=0$ such that $\left.\psi \circ h^{-1}\right|_{D \backslash\{0\}}=-\frac{\sin \theta}{r}$ in the polar coordinates $(r, \theta)$ associated with the disk $D$.

The 1-source-sink point is a mathematical expression for a uniform flow in $\mathcal{D}_{\zeta}(M)$ in terms of Hamiltonian vector fields and it induces a dipole-like singular streamline pattern locally in 
the neighborhood of the origin as shown in Figure 1(a), which consists of infinitely many selfconnecting orbits to the 1 -source-sink point. Note that each self-connecting streamline to the origin is mapped to a streamline of the uniform flow in $\mathcal{D}_{z}(M)$ by the conformal mapping.

When there is no 1-source-sink point, we may use the standard definition of structural stability with the $C^{r}$ topology given by Ma and Wang [11]. Then the set of Hamiltonian vector fields is denoted by $\mathcal{H}^{r}$. On the other hand, in the presence of the 1-source-sink point, we need to loosen the definition of structural stability, since the 1-source-sink point is structurally unstable by the standard definition of the structural stability. To be specific, we say that a vector field $V$ is a Hamiltonian vector field with a 1-source-sink point $p$, if $\left.V\right|_{\mathcal{D}_{\zeta}(M) \backslash\{p\}}$ is a Hamiltonian vector field on $\mathcal{D}_{\zeta}(M) \backslash\{p\}$. Let $\chi_{1}^{r}$ denote the set of $C^{r}$ Hamiltonian vector fields with a single 1-source-sink point on $\mathcal{D}_{\zeta}(M)$ with the $C^{r}$ topology $(r \geq 1)$. The definition of structural stability is given as follows[15].

Definition 2.2. For $s \leq r, V \in \chi_{1}^{r}$ is locally $\left(C^{s}\right)$-structurally stable at $p \in \mathcal{D}_{\zeta}(M)$ in $\chi_{1}^{r}$, if for any neighborhood $U$ of $p$ and any Hamiltonian vector field $\widetilde{V}$, which is $C^{s}$-near of $V$ in $\chi_{1}^{r}, \widetilde{V}$ is topologically equivalent to $V$. In other words, there is a homeomorphism $h: U \rightarrow h(U) \subset \mathcal{D}_{\zeta}(M)$ such that $h$ maps each orbit of $\left.V\right|_{U}$ to that of $\widetilde{V}$ homeomorphically and it preserves the orientation of the orbits.

Figure 1(b) shows the orbits that describe structurally stable Hamiltonian vector fields in $\mathcal{H}^{r}$ and $\chi_{1}^{r}$. A self-connecting orbit to the 1-source-sink point is called an ss-orbit. A nondegenerate hyperbolic stagnation point with homoclinic connections is a homoclinic saddle point. A streamline between a saddle point and the 1-source-sink point is called an ss-saddle connection. A $\partial$-saddle is a point of separation on a physical boundary. A streamline linking two $\partial$-saddles on the same boundary is called a $\partial$-saddle connection. A $\partial$-saddle connected to the 1-source-sink point is specially referred to as an $s s-\partial$-saddle and its connecting orbit is called an $s s$ - $\partial$-saddle connection. A periodic orbit is a closed streamline. According to $[11,15]$, the structurally stable Hamiltonian vector fields in $\mathcal{H}^{r}$ and $\chi_{1}^{r}$ are characterized using these orbits as follows.

Theorem 2.1 (Theorem 2.3.8. p.74 [11] and Theorem 3.2 [15]). A streamline pattern $V$ in $\mathcal{H}^{r}$ (resp. $\chi_{1}^{r}$ ) is structurally stable in $\mathcal{H}^{r}$ (resp. $\chi_{1}^{r}$ ) if and only if (S1) $V$ is regular, (S2) each saddle connection is self-connected, and (S3) each $\partial$-saddle connection is self-connected (i.e. the ends of each $\partial$-saddle connection belong to the same circular boundary).

This indicates that the streamline patterns of the structurally stable vector fields are topologically represented by non-degenerate hyperbolic and elliptic stagnation points, non-degenerate $\partial$-saddles, the 1-source-sink point and their connecting orbits. Topological streamline patterns composed of these orbits in $\mathcal{D}_{\zeta}(M)$ are called saddle connection diagrams for $\mathcal{H}^{r}$ and ss-saddle connection diagrams for $\chi_{1}^{r}$. Although there exist infinitely many ss-orbits and periodic orbits in the streamline patterns, for convenience, they are not shown in (ss-)saddle connection diagrams, since they do not affect the topological structure of streamlines. Moreover, the 1-source-sink point at the origin of $\mathcal{D}_{\zeta}(M)$ in $\chi_{1}^{r}$ is symbolized by (S) in ss-saddle connection diagrams. For example, the ss-saddle connection diagram for the streamline pattern of Figure 1(b) is shown in Figure 1(c).

Let us now explain how to assign a sequence of words to a given structurally stable Hamiltonian vector field. First, we introduce three fundamental structurally stable streamline patterns in $\mathcal{D}_{\zeta}(0)$ and $\mathcal{D}_{\zeta}(1)$ whose (ss-)saddle connection diagrams are shown in Figure 2. In the presence of the 1-source-sink point, there exist two fundamental streamline patterns in $\mathcal{D}_{\zeta}(0)$, which are referred to as Pattern $I$ in Figure 2(a) and Pattern $I I$ in Figure 2(b) respectively. Remembering that the indices of the 1 -source-sink point, a saddle point and a $\partial$-saddle are $2,-1$ and $-1 / 2$ respectively, 
there is no other structurally stable streamline pattern except for those two patterns, since they correspond to $1-M=2+(-1)$ and $1-M=2+2 \times(-1 / 2)$ in terms of the equalities of the Euler numbers for $M=0$. On the other hand, without the 1-source-sink point, we are unable to construct any streamline pattern in $\mathcal{D}_{\zeta}(0)$ and thus we give another fundamental pattern consisting of infinitely many periodic orbits in $\mathcal{D}_{\zeta}(1)$. We call this pattern Pattern $O$ whose saddle connection diagram is shown in Figure 2(c). Next, we define five operations that increase the genus element by one whilst maintaining structural stability. See Figure 3. The operation $A_{0}$ replaces an ss-orbit with a homoclinic saddle point connected to the 1-source-sink point with a pair of ss-saddle connections. By the operation $A_{2}$, we cut an ss-orbit and link a new circular boundary with the ss-orbit at the two cut points. The operations $B_{0}$ and $B_{2}$ replace a periodic orbit with a figure-eight pattern and a circular boundary with a $\partial$-saddle connection, respectively. The operation $C$ adds a new $\partial$-saddle connection to a circular boundary with more than one $\partial$-saddle. Note that the operations $A_{0}$ and $A_{2}$ (respectively $B_{0}$ and $B_{2}$ ) are applicable to ss-orbits (respectively periodic orbits) as long as they exist, while the operation $C$ is not applicable to any circular boundary without $\partial$-saddles.

In view of Theorem 2.1, any structurally stable Hamiltonian streamline pattern in $\mathcal{D}_{\zeta}(M)$ is constructed by applying the five operations repeatedly to either of the three patterns $I, I I$ and $O$. Accordingly, by arranging all operations applied to the patterns $I, I I$ and $O$ in a sequence, it is regarded as a word representation of this structurally stable streamline pattern. The sequences of operations starting from $I, I I$ and $O$ are called $I$-words, $I I$-words and $O$-words, respectively. It has been shown in [15] that any $I$-word, $I I$-word and $O$-word for structurally stable Hamiltonian vector fields have their own canonical expressions, called the maximal $I$-word, the maximal IIword and the maximal $O$-word. That is to say, let $W(s, t, u)=B_{0}^{s} B_{2}^{t} C^{u}$ for non-negative integers $s, t$ and $u$, denote a block component composed of $B_{0}, B_{2}$ and $C$. Then the canonical expressions of the maximal words are given as follows[15].

Theorem 2.2 (Theorem 3.3 of [15]). For any maximal $O$-word of a structurally stable streamline pattern in $\mathcal{D}_{\zeta}(M)$, there exist integers $k \geq 1, s_{m}, t_{m} \geq 0$ for $m=1, \ldots, k$ and $u_{m}>0$ for $m=1, \ldots, k-1$ such that it is expressed by

$$
O W\left(s_{1}, t_{1}, u_{1}\right) \cdots W\left(s_{k-1}, t_{k-1}, u_{k-1}\right) W\left(s_{k}, t_{k}, 0\right)
$$

where $t_{m}>0$ for any $m<k$ with $M=1+\sum_{m=1}^{k-1}\left(s_{m}+t_{m}+u_{m}\right)+s_{k}+t_{k}$.

Theorem 2.3 (Theorem 3.4 of [15]). Let $p, q, r$ be non-negative integers. Then, for any maximal $I$-word of a structurally stable streamline pattern in $\mathcal{D}_{\zeta}(M)$, there exist integers $k \geq 1, s_{m}, t_{m} \geq 0$ for $m=1, \ldots, k$ and $u_{m}>0$ for $m=1, \ldots, k-1$ such that it is expressed by

$$
I\left(A_{0}\right)^{p}\left(A_{2}\right)^{q}(C)^{r} W\left(s_{1}, t_{1}, u_{1}\right) \cdots W\left(s_{k-1}, t_{k-1}, u_{k-1}\right) W\left(s_{k}, t_{k}, 0\right),
$$

where $t_{m}>0$ for any $m<k$ with $M=p+q+r+\sum_{m=1}^{k-1}\left(s_{m}+t_{m}+u_{m}\right)+s_{k}+t_{k}$, if $p+r>0$. Otherwise it is represented by $I\left(A_{2}\right)^{q}$ with $M=q$.

Theorem 2.4 (Theorem 3.5 of [15]). Let $p$ be a non-negative integer. Then, for any maximal $I I$-word of a structurally stable streamline pattern in $\mathcal{D}_{\zeta}(M)$, there exist integers $k \geq 1, s_{m}, t_{m} \geq 0$ for $m=1, \ldots, k$ and $u_{m}>0$ for $m=1, \ldots, k-1$ such that it is expressed by

$$
I I\left(A_{0}\right)^{p} W\left(s_{1}, t_{1}, u_{1}\right) \cdots W\left(s_{k-1}, t_{k-1}, u_{k-1}\right) W\left(s_{k}, t_{k}, 0\right),
$$

where $t_{m}>0$ for any $m<k$ with $M=p+\sum_{m=1}^{k-1}\left(s_{m}+t_{m}+u_{m}\right)+s_{k}+t_{k}$. 
The uniqueness of the maximal word representation has been discussed in [13], where it has been shown that every maximal word represents a set of structurally stable Hamiltonian vector fields, while each structurally stable Hamiltonian vector field has a unique maximal word representation. Moreover, in [13], encoding algorithms providing the unique maximal word for a given structurally stable Hamiltonian vector field are presented. This allows us to label any given structurally stable streamline pattern uniquely with its maximal word representation.

Now we are ready to state the purpose of the present study. See Figure 4. The streamline pattern in Figure 4(b) is structurally unstable, since there exist arbitrarily small continuous perturbations which give rise to two structurally stable streamline patterns represented by the maximal words $I A_{0} A_{2}$ and $I A_{0} B_{2}$, shown in Figure 4(a) and (c). In other words, the unstable streamline pattern in Figure $4(\mathrm{~b})$ is the transient state between the two structurally stable patterns represented by $I A_{0} A_{2}$ and $I A_{0} B_{2}$. The question we consider in this paper is whether it is possible to determine the transient streamline pattern in Figure 4(b) from the two maximal words $I A_{0} A_{2}$ and $I A_{0} B_{2}$ conversely. The present study provides a procedure to identify all possible transitions between two structurally stable Hamiltonian vector fields by comparing their maximal words and their corresponding streamline topologies. In order to do this, we introduce the indices and subwords of the maximal words as follows.

Definition 2.3. Let $a_{0}(W), a_{2}(W), b_{0}(W), b_{2}(W)$ and $c(W)$ denote the number of words $A_{0}, A_{2}$, $B_{0}, B_{2}$ and $C$ contained in the maximal word $W$, respectively. Then the indices for the maximal $O$-word, the maximal $I$-word and the maximal II-word $W$ are defined by, respectively, as

$$
\begin{aligned}
\operatorname{ind}_{O}(W) & =\left(0,0, b_{0}(W), b_{2}(W), c(W)\right), \\
\operatorname{ind}_{I}(W) & =\left(a_{0}(W), a_{2}(W)+1, b_{0}(W), b_{2}(W), c(W)\right), \\
\operatorname{ind}_{I I}(W) & =\left(a_{0}(W)+1, a_{2}(W), b_{0}(W), b_{2}(W), c(W)\right) .
\end{aligned}
$$

Definition 2.4. For a given maximal word $W=O_{0} O_{1} \ldots O_{k}$, where $O_{0} \in\{O, I, I I\}$ and $O_{i}$ $(1 \leq i \leq k)$ are either $A_{0}, A_{2}, B_{0}, B_{2}$ or $C$, a sub sequence of $W$, say $S=O_{i_{1}} \ldots O_{i_{l}}$ for $0 \leq i_{1}<\cdots<i_{l} \leq k$, is called the subword of $W$, which is symbolized by $S \subseteq W$.

A subword $S \subseteq W$ is a subsequence $S$ contained in the maximal word $W$. Note that $i_{1}=0$ is permitted in this definition, which indicates that the header of the maximal word, namely $O, I$ or $I I$, can be contained in the subword.

We introduce some orbits that describe structurally unstable streamline patterns. A streamline connecting two different saddle points is called a heteroclinic orbit. See Figure 5(a). An $s$ - $\partial$ saddle connection links a saddle point with a $\partial$-saddle on a circular boundary as illustrated in Figure 5(b). We call an orbit a heteroclinic $\partial$-orbit when it connects $\partial$-saddles on two different circular boundaries as shown in Figure 5(c). These heteroclinic orbits induce regular Hamiltonian vector fields, although they are structurally unstable in the sense that they do not satisfy (S2) or (S3) in Theorem 2.1. By a heteroclinic pair, we mean either two saddle points connected by heteroclinic orbits (Figure 5(a)), a pair of a saddle point and a circular boundary connected by $s$ $\partial$-saddle connections (Figure $5(\mathrm{~b})$ ), or two circular boundaries connected by heteroclinic $\partial$-saddle connections (Figure 5(c)). We then define an important class of structurally unstable Hamiltonian vector fields.

Definition 2.5. A regular Hamiltonian vector field is called h-unstable (or it is an h-unstable streamline pattern) if it has exactly one heteroclinic pair.

On the other hand, a stagnation point on a circular boundary is called a pinching point if it is a degenerate saddle on a boundary with four separatrices. A homoclinic pinching orbit is 
a streamline that connects a pinching point homoclinically as shown in Figure $5(\mathrm{~d})$. We then introduce another class of structurally unstable Hamiltonian vector fields as follows.

Definition 2.6. A non-regular Hamiltonian vector field is called p-unstable (or it is a p-unstable streamline pattern) if it has just one pinching point but no heteroclinic pair and it is regular except this pinching point.

Any vector field with a pinching point is structurally unstable, since the point is a degenerate stagnation point that does not satisfy (S1) in Theorem 2.1. We use structurally $h$-unstable and $p$-unstable vector fields to describe transient streamline patterns between structurally stable streamline patterns, since the set of $h$-unstable and $p$-unstable Hamiltonian vector fields is open dense in all structurally unstable Hamiltonian vector fields in $\mathcal{D}_{\zeta}(M)$ for every fixed $M$ under suitable conditions as discussed in $\S 3$.

\section{Structurally unstable Hamiltonian vector fields}

According to Theorem 2.1, structurally unstable Hamiltonian vector fields do not satisfy either of the three conditions (S1), (S2) or (S3). We first characterize unstable vector fields that are not regular, when (S1) is not satisfied. Let us recall that a vector field $v$ in $\mathcal{H}^{r}$ or $\chi_{1}^{r}$ is called regular if all stagnation points except the 1-source-sink point are non-degenerate. That is to say, the Jacobian of the derivative of $v$, i.e. the Hessian of the Hamiltonian, is non-zero. Generally, since non-regular vector fields can have various singular structures, we impose some restrictions on the set of structurally unstable vector fields for the sake of simplicity as follows.

Suppose first that we consider Hamiltonian vector fields on compact connected domains with a finite number of stagnation points in this paper. Then, Cobo et al.[9] have shown that they have topological centers, $k$-saddles and $k / 2$ - $\partial$-saddles. A stagnation point $p$ is called a topological center, if there is a neighborhood of $p$ where all orbits except $p$ are periodic. Note that all topological centers do not need to be degenerate, since an elliptic fixed point is a regular topological center. On the other hand, for an integer $k \geq 0$, a stagnation point on a boundary with $k+2$ separatrices is called a $k / 2$ - $\partial$-saddle, while a stagnation point located in the interior of $\mathcal{D}_{\zeta}(M)$ with $2 k+2$ separatrices is said to be a $k$-saddle. See Figure 6 . Note that non-degenerate $\partial$-saddles and hyperbolic saddle points are $1 / 2$ - $\partial$-saddles and 1-saddles, respectively.

As are non-degenerate elliptic fixed points, degenerate topological centers in streamline patterns are also regarded as genus elements in the multiply connected domain $\mathcal{D}_{\zeta}(M)$. Therefore, if the emergence of topological centers is permitted, the perturbation of degenerate stagnation points yields new topological centers, i.e. genus elements, in their neighborhood as shown in Figure $7(\mathrm{a})$ and (b). We thus rule out the existence of degenerate topological centers and we would like to use structurally unstable Hamiltonian vector fields to describe transient states between two different structurally stable vector fields with the same number of genus elements. Furthermore, we may assume non-existence of 0 - $(\partial$-) saddle, degenerate $1 / 2$ - $\partial$-saddles, degenerate 1 -saddles in structurally unstable vector fields for the following reasons. First, small perturbations of degenerate $0-(\partial-)$ saddles give rise to new topological centers, which is now prohibited. Second, regular 0 - $\partial$-saddles don't contribute to transitions, since any small perturbation near them does not change (ss-)saddle connection diagrams. Third, even if there exist degenerate $1 / 2$ - $\partial$-saddles and degenerate 1-saddles, we are unable to distinguish the streamline structures around these degenerate points from those with non-degenerate $\partial$-saddles and non-degenerate hyperbolic saddles, since structurally stable streamline patterns are determined by 1 -saddles and $1 / 2$ - $\partial$-saddles up to topological equivalence. 
It follows from the observation above that that we consider the set of structurally unstable Hamiltonian vector fields in $\mathcal{H}^{r}$ (or $\chi_{1}^{r}$ respectively) composed of a finite number of non-degenerate stagnation points, degenerate $k$-saddles and degenerate $k / 2$ - $\partial$-saddles for $k \geq 2$, which is denoted by $\mathcal{H}^{u n}$ (or $\chi_{1}^{u n}$ respectively). Here, we look at these sets in detail. Let $H(x, y)$ be a Hamiltonian defined in the neighborhood of a stagnation point at the origin of $\mathbb{R}^{2}$. Suppose that the origin is a degenerate stagnation point, namely the Hessian of $H$ at the origin has at least one zero eigenvalue. If the Hessian has only one zero eigenvalue, the Hamiltonian is represented by $H(x, y)=a_{0} x^{2}+$ $O(3)$ for a non-zero real $a_{0}$ under a certain linear transformation, in which $O(3)$ symbolizes the higher order terms of degree 3 . Then the degenerate origin becomes a 1-saddle, since the $x$-axis becomes a separatrix and the existence of another separatrix is assured by the divergence free condition. Hence, the Hamiltonian vector field does not belong to $\mathcal{H}^{u n}$ or $\chi_{1}^{u n}$. If the Hessian has two zero eigenvalues, the Hamiltonian contains either of the following leading order terms of degree 3:

(Case 1) For reals $a_{1} \neq 0, b_{1} \neq b_{1}^{\prime} \neq b_{1}^{\prime \prime}, H(x, y)=a_{1}\left(x-b_{1} y\right)\left(x-b_{1}^{\prime} y\right)\left(x-b_{1}^{\prime \prime} y\right)+O(4)$. The degenerate origin becomes a 2 -saddle.

(Case 2) For reals $a_{2} \neq 0, b_{2}>0$ and $b_{2}^{\prime}, H(x, y)=a_{2}\left(x^{2}+b_{2} y^{2}\right)\left(x-b_{2}^{\prime} y\right)+O(4)$. The degenerate origin becomes a 0 -saddle.

(Case 3) For reals $a_{3} \neq 0, b_{3} \neq b_{3}^{\prime}, H(x, y)=a_{3}\left(x-b_{3} y\right)^{2}\left(x-b_{3}^{\prime} y\right)+O(4)$. The degenerate origin becomes a 1 -saddle.

(Case 4) For reals $a_{4} \neq 0, b_{4}, H(x, y)=a_{4}\left(x-b_{4} y\right)^{3}+O(4)$, which yields a 1-saddle structure at the degenerate origin owing to the divergence free condition.

Since degenerate 0 -saddles and 1 -saddles are not contained in $\mathcal{H}^{\text {un }}$ or $\chi_{1}^{\text {un }}$, it is sufficient to consider the first case only. To generalize, suppose that the terms of degree $k-1(k \geq 4)$ in the Hamiltonian vanish and it is represented by $H=c_{k} \prod_{m=1}^{k}\left(x-d_{m} y\right)+O(k+1)$ for reals $c_{k} \neq 0$ and $d_{i} \neq d_{j}(i \neq j)$. Then, the degenerate origin becomes a $(k-1)$-saddle. However, $(k-1)$ saddles for $k \geq 4$ are non generic in $\mathcal{H}^{u n}$ and $\chi_{1}^{u n}$, since an arbitrarily small perturbation of the Hamiltonian gives rise to the terms of degree three immediately, in which the stagnation points become 2 -saddles. With a similar argument, we can show that the 1 - $\partial$-saddles exist generically in $\mathcal{H}^{u n}$ and $\chi_{1}^{u n}$. Hence, we conclude that structurally unstable Hamiltonian vector fields whose degenerate stagnation points are 2 -saddles and degenerate 1 - $\partial$-saddles are dense in $\mathcal{H}^{\text {un }}$ and $\chi_{1}^{\text {un }}$.

Suppose now that a vector field $v$ is regular but does not satisfy (S2) or (S3). Then it has a finite number of heteroclinic pairs. When we fix one of the heteroclinic pairs, an arbitrarily small perturbation near $(\partial$-)saddles in the other heteroclinic pairs yields non-degenerate homoclinic $(\partial-)$ saddles as shown in the proof of Theorem 2.1 of [15]. This means that $v$ is approximated in the $C^{r}$ topology by $h$-unstable vector fields. On the other hand, if a vector field $v$ does not satisfy (S1), it follows from the argument above that $v$ becomes a structurally unstable vector field with degenerate 2 -saddles and degenerate 1 - $\partial$-saddles. Fixing one of the 2 -saddles, we can make the other 2 -saddles and $1-\partial$-saddles into non-degenerate stagnation points by an arbitrarily small perturbation. Furthermore, the 2 -saddle is perturbed in the $C^{r}$ topology by a heteroclinic saddle connection as shown in Figure 8. On the other hand, since the 1 - $\partial$-saddle is a pinching point, we obtain a sequence of $p$-unstable vector fields that converges to the degenerate vector field by holding one of the 1 - $\partial$-saddles and perturbing the 2 -saddles and the other 1 - $\partial$-saddles. Consequently, we have the following proposition.

Proposition 3.1. For every fixed number of genus elements, the set of h-unstable and p-unstable Hamiltonian vector fields with the same number of genus elements is open dense in $\mathcal{H}^{\text {un }}$ and $\chi_{1}^{u n}$. 
Note that the set of $h$-unstable or $p$-unstable Hamiltonian vector field is a co-dimension one subspace of the set of $2 \mathrm{D}$ Hamiltonian vector fields, through which generic transitions between two structurally stable streamline patterns with the same number of genus elements occur.

\section{Streamline patterns with a homoclinic pair}

\subsection{Operations increasing the genus element by one}

Let us define a series of operations that construct $h$-unstable streamline patterns in $\mathcal{D}_{\zeta}(M)$ from structurally stable streamline patterns in $\mathcal{D}_{\zeta}(M-1)$ by increasing the genus elements by one. Following this definition, we describe how these $h$-unstable streamline patterns are perturbed in $\mathcal{H}^{r}$ or $\chi_{1}^{r}$ and observe how their corresponding subwords and indices change as a result of the perturbation. For the sake of a simple description of the operations, every genus element is represented by a circular boundary in the following (ss-)saddle connection diagrams, but recall that any circular boundary without $\partial$-saddles can always be replaced by an elliptic fixed point or an isolated vortex structure like a point vortex topologically.

(Operations $D_{0, s}$ and $D_{0}$ ) By the operations $D_{0, s}$ and $D_{0}$, we append a homoclinic saddle enclosing a new genus element to an ss- $(\partial$-)saddle connection and a $\partial$-saddle connection respectively as shown in Figure 9(a). The lower suffix $s$ of $D_{0, s}$ indicates that the new structure is directly connected to the 1-source-sink point in the streamline patterns.

The operation $D_{0, s}$ is applicable to the fundamental patterns $I, I I$ and the structurally stable orbits created by the operations $A_{0}$ and $A_{2}$, since they have ss- $(\partial$-)saddle connections. Applying the operation $D_{0, s}$ to an ss- $\partial$-saddle connection in the pattern $I$, we have two $h$-unstable patterns $I D_{0, s}$, since we can append a homoclinic saddle in the upper or lower side of the ss- $\partial$-saddle connection. Perturbation of these patterns in $\chi_{1}^{r}$ yields two structurally stable patterns with the same word $I A_{0}$ as shown in Figure 10(a). Since the pattern $I I$ contains a pair of ss-saddle connections, we have the $h$-unstable patterns $I I D_{0, s}$, which are marginal states between the two stable patterns represented by the same word $I I A_{0}$. See Figure $10(\mathrm{~b})$. Since the operations $A_{0}$ and $A_{2}$ generate a pair of ss-saddle connections and ss- $\partial$-saddle connections respectively, the application of $D_{0, s}$ to these orbits yields the $h$-unstable patterns $A_{0} D_{0, s}$ in Figure 10 (c) and $A_{2} D_{0, s}$ in Figure 10(d). Under the perturbation in $\chi_{1}^{r}$, the $h$-unstable patterns $A_{0} D_{0, s}$ are reduced to the same patterns represented by $A_{0} A_{0}$. The streamline patterns $A_{2} D_{0, s}$ are topologically equivalent to $I D_{0, s}$ as shown in Figure 10(d).

As we observe here, the operation $D_{0, s}$ generates many structurally unstable patterns represented by the same symbol. For instance, we obtain three topologically different unstable patterns $A_{0} D_{0, s}$ depending on which direction we append a new homoclinic saddle. A great deal of space is required to document all such possible streamline patterns. On the other hand, notice that their perturbed structurally stable patterns are also represented by the same (sub)word. Hence, in the following illustrations, we show one of these patterns as their representative to avoid this redundancy.

The operation $D_{0}$ is applicable to a $\partial$-saddle connection generated by either the operation $B_{2}$ or $C$. Figure 11 (a) shows the $h$-unstable streamline pattern $B_{2} D_{0}$, whose perturbation induces a transition between the two different structurally stable patterns represented by the same word $B_{0} B_{2}$ (or $B_{2} B_{0}$ ). On the other hand, since the operation $C$ is applicable to circular boundaries with at least two $\partial$-saddles, we need to take the following two cases into considerations; (1) The two $\partial$-saddles lead to the 1 -source-sink point with a pair of ss- $\partial$-saddle connections; (2) They are 
connected together via a $\partial$-saddle connection. In the first case, the $h$-unstable streamline pattern is represented by $A_{2} C D_{0}$, which is topologically equivalent to the pattern $I C D_{0}$ as illustrated in Figure 11(b). Its perturbation in $\chi_{1}^{r}$ induces a transition between the two different structurally stable patterns $I A_{0} C$ and $I C B_{0}$. In addition, if a $\partial$-saddle connection created by another operation $C$ encloses the $h$-unstable structure $C D_{0}$ with a pair of the s- $\partial$-saddle connections, which is represented by $I C C D_{0}$, its perturbation yields two different structurally stable patterns with the same word $I C C B_{0}$ (or $I C B_{0} C$ ) as illustrated in Figure 11(c). In the second case, we have the $h$ unstable pattern $B_{2} C D_{0}$ that is a transient state between the two streamline patterns represented by the same word $B_{2} C B_{0}$ (or $B_{0} B_{2} C, B_{2} B_{0} C$ ) as in Figure $11(\mathrm{~d}$ ). We can also consider the streamline pattern $B_{2} C D_{0}$ in Figure 11(e) where the $h$-unstable structure corresponding to $C D_{0}$ is enclosed by a $\partial$-saddle connection similarly as in the first case. Its perturbed patterns, which are shown in Figure 11(e), are represented by the same word $B_{2} C B_{0}$ (or $B_{0} B_{2} C, B_{2} B_{0} C$ ). This indicates that the same transition of words as $B_{2} C D_{0}$.

The above observations reveal that the perturbation of the $h$-unstable patterns created by $D_{0, s}$ and $D_{0}$ brings us the structurally stable streamline patterns with the same word representations except the $h$-unstable pattern $I C D_{0}\left(\approx A_{2} C D_{0}\right)$, whose perturbation gives rise to the change of the index $\operatorname{ind}_{I}\left(I A_{0} C\right)-\operatorname{ind}_{I}\left(I C B_{0}\right)=(1,0,-1,0,0)$.

(Operations $E_{0, s}$ and $E_{0}$ ) The operations $E_{0, s}$ and $E_{0}$ add a homoclinic saddle point enclosing a new genus element to a homoclinic saddle connection as shown in Figure 9(b). Since the fundamental pattern $I I$ contains a homoclinic saddle connection, the $h$-unstable pattern $I I E_{0, s}$ is illustrated in Figure 12(a). Perturbing the pattern in $\chi_{1}^{r}$, we have the two structurally stable patterns $I I A_{0}$ and $I I B_{0}$. Then the difference between their indices is given by $\operatorname{ind}_{I I}\left(I I A_{0}\right)-$ $\operatorname{ind}_{I I}\left(I I B_{0}\right)=(1,0,-1,0,0)$. Applying $E_{0, s}$ to a homoclinic saddle connection generated by the operation $A_{0}$, we have the $h$-unstable pattern $A_{0} E_{0, s}$, which splits into the two structurally stable patterns represented by $A_{0} A_{0}$ and $A_{0} B_{0}$ as shown in Figure 12(b). Since the streamline pattern $A_{0} E_{0, s}$ can be a subword of maximal $I$-words and maximal $I I$-words, their corresponding indices

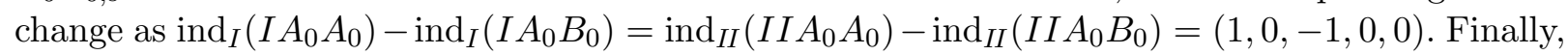
the operation $E_{0}$ is applicable only to a homoclinic saddle connection generated by the operation $B_{0}$ and thus we have the $h$-unstable streamline pattern $B_{0} E_{0}$ in Figure 12(c), which is perturbed into the pattern $B_{0} B_{0}$.

(Operations $\Phi_{0, s}$ and $\Phi_{0}$ ) Let $\Phi_{0, s}$ and $\Phi_{0}$ denote the operations splitting a homoclinic saddle point into two saddle points and connecting them with two new heteroclinic saddle connections enclosing a new genus element. These operations create a $\Phi$-shaped streamline pattern as illustrated in Figure 9(c).

Since the operations are applicable to the same structurally stable streamline patterns as $E_{0, s}$ and $E_{0}$, we have the three $h$-unstable streamline patterns $I I \Phi_{0, s}, A_{0} \Phi_{0, s}$ and $B_{0} \Phi_{0}$. Figure 13 (a) shows the $h$-unstable pattern $I I \Phi_{0, s}$ and its perturbed structurally stable pattern $I I A_{0}$. The $h$ unstable pattern $A_{0} \Phi_{0, s}$ in Figure 13(b) is a transient state between the structurally stable patterns $A_{0} A_{0}$. The $h$-unstable pattern $B_{0} \Phi_{0}$ is also perturbed into the stable streamline patterns $B_{0} B_{0}$ as shown in Figure 13(c). Hence, the perturbation of the $h$-unstable patterns generated by $\Phi_{0, s}$ and $\Phi_{0}$ never change their word representations.

(Operations $D_{2, s}$ and $D_{2}$ ) When we cut an ss- $(\partial$-)saddle connection (respectively a $\partial$-saddle connection) and connect a new circular boundary at the two cut points, the operation is symbolized by $D_{2, s}$ (respectively $D_{2}$ ) as shown in Figure $9(\mathrm{~d})$. The lower suffix 2 of the operations $D_{2, s}$ and 
$D_{2}$ means that they create two $\partial$-saddles at the new circular boundary. They are applicable to the same structurally stable patterns as the operations $D_{0, s}$ and $D_{0}$.

In $\chi_{1}^{r}$, the $h$-unstable pattern $I D_{2, s}$ is perturbed into the two structurally stable patterns represented by $I A_{2}$ in Figure 14(a). When we perturb the $h$-unstable pattern $I I D_{2, s}$ in $\chi_{1}^{r}$, we have the stable patterns $I I A_{2}$ that are topologically equivalent to $I A_{0}$. In this case, the word representations of the $h$-unstable pattern and its perturbed structurally stable patterns have the different headers, whereas their indices remain the same. See Figure 14(b). Applying the operation $D_{2, s}$ to an ss-saddle connection created by the operation $A_{0}$, we obtain the $h$-unstable streamline pattern $A_{0} D_{2, s}$. It is topologically equivalent to $A_{2} D_{0, s}$ in Figure $14(\mathrm{c})$ that has already been discussed. Similarly, it is unnecessary to consider the $h$-unstable pattern $A_{2} D_{2, s}$, since it is equivalent to $I D_{2, s}$ as shown in Figure 14(d).

The operation $D_{2}$ is applicable to a $\partial$-saddle connection created by the operations $B_{2}$ and $C$. The perturbation of the $h$-unstable pattern $B_{2} D_{2}$ yields the same structurally stable pattern $B_{2} B_{2}$ as illustrated in Figure 15(a). In a similar manner to that discussed with regards to the operator $D_{0}$, we need to consider the following $h$-unstable patterns $A_{2} C D_{2} \approx I C D_{2}$ in Figure $15(\mathrm{~b})$, $I C C D_{2}$ in Figure 15(c), $B_{2} C D_{2}$ in Figure 15(d) and $B_{2} C C D_{2}$ in Figure 15(e). The perturbation of the $h$-unstable pattern $I C D_{2}$ brings us two different structurally stable patterns represented by $I A_{2} C$ and $I C B_{2}$, which result in the change of index, $\operatorname{ind}_{I}\left(I A_{2} C\right)-\operatorname{ind}_{I}\left(I C B_{2}\right)=(0,1,0,-1,0)$. Perturbation of the patterns $I C C D_{2}$ and $B_{2} C D_{2}$ induce transitions between the two different structurally stable patterns represented by the same word $I C C B_{2}$ (or $I C B_{2} C$ ) and $B_{2} C B_{2}$ (or $B_{2} B_{2} C$ ) respectively and thus no change of index occurs. The last pattern $B_{2} C C D_{2}$ in Figure $15(\mathrm{~d})$ is obtained by applying $C$ to the pattern $B_{2} C D_{2}$. Perturbing this pattern yields two structurally stable patterns that are different from those shown in Figure 15(d) but their word representation is equivalent to $B_{2} C B_{2}$ with additional $C$ being applied. Thus the pattern $B_{2} C C D_{2}$ induces the same transition of the maximal words as the pattern $B_{2} C D_{2}$.

(Operations $E_{2, s}$ and $E_{2}$ ) Let $E_{2, s}$ and $E_{2}$ denote the operations cutting a homoclinic saddle connection and connecting a new circular boundary at the two cut points as shown in Figure 9(e). These operations are applicable to the same orbits as $E_{0, s}$ and $E_{0}$, but they create new two $\partial$-saddles on the circular boundary.

The $h$-unstable streamline pattern $I I E_{2, s}$ is a transient state between the structurally stable patterns $I I A_{2}=I A_{0}$ and $I I B_{2}$ as shown in Figure 16(a). With the perturbation in $\chi_{1}^{r}$, we have the change of header between the maximal $I$-words and the maximal $I I$-words and the change of index, $\operatorname{ind}_{I}\left(I A_{0}\right)-\operatorname{ind}_{I I}\left(I I B_{2}\right)=(0,1,0,-1,0)$. Applying $E_{2, s}$ to a homoclinic saddle connection generated by the operation $A_{0}$, we have the $h$-unstable pattern $A_{0} E_{2, s}$. Perturbing this pattern gives rise to different transitions between structurally stable patterns depending on whether the pattern represented by $A_{0} E_{2, s}$ is contained in the fundamental pattern $I$ or $I I$. The $h$-unstable streamline pattern $I A_{0} E_{2, s}$ splits into the structurally stable patterns $I A_{0} A_{2}$ and $I A_{0} B_{2}$ as illustrated in Figure 16(b). The transition yields the change of index, $\operatorname{ind}_{I}\left(I A_{0} A_{2}\right)-$ $\operatorname{ind}_{I}\left(I A_{0} B_{2}\right)=(0,1,0,-1,0)$. On the other hand, the $h$-unstable streamline pattern $I I A_{0} E_{2, s}$ is topologically equivalent to $I I E_{2, s} A_{0}$ as shown in Figure 16(c). Finally, the operation $E_{0}$ is applicable only to a homoclinic saddle connection generated by the operation $B_{0}$, but the $h$ unstable pattern $B_{0} E_{2}$ is topologically equivalent to the $h$-unstable pattern $B_{2} D_{0}$, which has already been discussed. 


\subsection{Operations increasing the genus elements by more than one}

We introduce more operations creating $h$-unstable streamline patterns with increasing the genus elements by more than one to structurally stable streamline patterns. See Figure 17.

(Operations $\Psi_{s}(2)$ and $\Psi(2)$ ) Picking up a $\partial$-saddle on a circular boundary, we add a new saddle point on the orbit connecting the $\partial$-saddle and the new two $\partial$-saddles on the same circular boundary, and we then connect the saddle point and the two $\partial$-saddles with s- $\partial$-saddle connections. These operations create a $\Psi$-shaped streamline pattern as illustrated in Figure 17(a). When it is applied to a $\partial$-saddle connected to the 1 -source-sink point, the operation is denoted by $\Psi_{s}(2)$. Otherwise we symbolize the operation as $\Psi(2)$. These operations increase the genus elements by two enclosed by the three s- $\partial$-saddle connections. The increased number is explicitly expressed in the parentheses after the symbols $\Psi_{s}$ and $\Psi$.

A $\partial$-saddle to which the operations are applicable exists in the fundamental pattern $I$ and the streamlines generated by $A_{2}, B_{2}$ and $C$. Figure 18(a) shows the $h$-unstable streamline pattern represented by $I \Psi_{s}(2)$, which is perturbed into the structurally stable patterns represented by the same word $I A_{0} C$. The $h$-unstable pattern $A_{2} \Psi_{s}(2)$ is topologically equivalent to $I \Psi_{s}(2)$ as shown in Figure 18(b).

Applying the operation $\Psi(2)$ to a $\partial$-saddle created by the operation $B_{2}$, we obtain the $h$ unstable pattern $B_{2} \Psi(2)$ in Figure 18(c). By perturbation in $\mathcal{H}^{r}$ or $\chi_{1}^{r}$, this pattern splits into the two different structurally stable patterns with the same word representation $B_{0} B_{2} C$ (or $B_{2} C B_{0}$, $\left.B_{2} B_{0} C\right)$. When a circular boundary with a $\partial$-saddle has a pair of ss- $\partial$-saddle connections, we have the $h$-unstable pattern $I C \Psi(2)$ as shown in Figure 19(a). Perturbing this pattern in $\chi_{1}^{r}$ results in a transition between the two structurally stable patterns represented by $I A_{0} C C$ and $I C C B_{0}$ (or $\left.I C B_{0} C\right)$, and the change of index, $\operatorname{ind}_{I}\left(I A_{0} C C\right)-\operatorname{ind}_{I}\left(I C C B_{0}\right)=(1,0,-1,0,0)$. Figure 19(b) shows the perturbation of the $h$-unstable streamline represented by $I C C \Psi(2)$, where the structure corresponding to $C \Psi(2)$ is enclosed by a $\partial$-saddle connection. This pattern is perturbed into different structurally stable patterns represented by the same word $I C C C B_{0}$ (or $I C C B_{0} C$, $\left.I C B_{0} C C\right)$. On the other hand, if a circular boundary with a $\partial$-saddle has no connection with the 1-source-sink point, the $h$-unstable pattern $B_{2} C \Psi(2)$ induces a transition between different stable patterns represented by the same word $B_{2} C C B_{0}$ (or $B_{0} B_{2} C C, B_{2} B_{0} C C, B_{2} C B_{0} C$ ) as shown in Figure 19(c). When the streamline pattern corresponding to $C \Psi(2)$ is enclosed by a $\partial$-saddle connection as shown in Figure 19(d), it is equivalent to the pattern represented by $B_{2} C \Psi(2) C$. Its perturbed patterns are different from those in Figure 19(c), but we have the same transition of the maximal words as $B_{2} C \Psi(2)$.

(Operations $\Xi_{s}(2 n-1)$ and $\left.\Xi(2 n-1), n \geq 2\right)$ By the operations $\Xi_{s}(2 n-1)$ and $\Xi(2 n-1)$, we add any positive odd numbers of circular boundaries. To be specific, for a circular boundary with a pair of $\partial$-saddles, we add a new circular boundary with a $\partial$-saddle and then connect the two circular boundaries with $2 n-1$ heteroclinic $\partial$-orbits. See Figure 17(b). As a result of these operations, $2 n-1$ genus elements and $2 \times(2 n-1) \partial$-saddles on the circular boundaries are created. Note that the operations are the same as $D_{2}$ and $D_{2, s}$ for $n=1$ and thus we assume $n \geq 2$ without loss of generality.

The streamlines to which the operations $\Xi_{s}(2 n-1)$ and $\Xi(2 n-1)$ are applicable are the same as those of $\Psi_{s}(2)$ and $\Psi(2)$. The $h$-unstable pattern $I \Xi_{s}(2 n-1)$ is a transient state between the structurally stable patterns represented by $I A_{0} C^{2 n-2}$ in Figure 20(a). The $h$-unstable pattern $A_{2} \Xi_{s}(2 n-1)$ is excluded, since we have the equivalence $A_{2} \Xi_{s}(2 n-1) \approx I \Xi_{s}(2 n-1)$. Regarding 
the $h$-unstable pattern $B_{2} \Xi(2 n-1)$, its perturbed stable pattern remains the same and its word representation is $B_{2} C^{k} B_{2} C^{2 n-2-k}$ for $0 \leq k \leq n-1$ as shown in Figure 20(b).

The operation $\Xi(2 n-1)$, when it is applied to a circular boundary with a pair of ss- $\partial$-saddle connections and a $\partial$-saddle connection created by the operation $C$, yields the $h$-unstable pattern $I C \Xi(2 n-1)$ as shown in Figure 20(c). The perturbation of this pattern brings us a transition between the structurally stable patterns $I A_{2} C^{2 n-1}$ and $I C^{k} B_{2} C^{2 n-1-k}$ for $1 \leq k \leq n$, for which the following change of index occurs:

$$
\operatorname{ind}_{I}\left(I A_{2} C^{2 n-1}\right)-\operatorname{ind}_{I}\left(I C^{k} B_{2} C^{2 n-1-k}\right)=(0,1,0,-1,0) .
$$

If the circular boundary with multiple heteroclinic connections is enclosed by another $\partial$-saddle connection whose streamline pattern is represented by $\operatorname{ICC} \Xi(2 n-1)$, as shown in Figure $20(\mathrm{~d})$, the perturbation of this unstable pattern yields the two different structurally stable patterns with the same word $I C^{k} B_{2} C^{2 n-k}$ for $1 \leq k \leq n$. Finally, it is unnecessary to deal with the $h$-unstable pattern $B_{2} C \Xi(2 n-1)$, since it is equivalent to $B_{2} \Xi(2 n-1) C$.

\section{3 $H$-unstable streamline patterns with heteroclinic connections}

We show that there exists no operation that generates $h$-unstable streamline patterns with heteroclinic connections except the fourteen operations defined in $\S \S 4.1$ and 4.2. Let us first remember the following three facts.

(i) Any heteroclinic orbit links a heteroclinic pair.

(ii) Any non-degenerate saddle point has four connecting orbits. Each connecting orbit leads either to the 1-source-sink point, or to the same saddle point homoclinically unless it is connected with a different saddle point or a different $\partial$-saddle heteroclinically.

(iii) Any circular boundary in structurally stable streamline patterns has a non-negative even number of $\partial$-saddles. Each connecting orbit from a $\partial$-saddle leads to the 1 -source-sink point or another $\partial$-saddle on the same boundary unless it leads to a saddle point or a $\partial$-saddle on a different boundary heteroclinically.

The fact (i) tells us that a heteroclinic orbit either connects two different non-degenerate saddle points, a non-degenerate saddle point and a $\partial$-saddle, or $\partial$-saddles on different circular boundaries. Each of these cases is now discussed.

First, we suppose that heteroclinic orbits link two different saddle points. When only one heteroclinic orbit connects these points, there remain three free connecting orbits for each saddle point. According to (ii), two of them form a homoclinic saddle connection and the one free connecting orbit leads to the 1 -source-sink point. Otherwise, it forms another heteroclinic connection, which contradicts the assumption. We then have the $h$-unstable pattern generated by the operation $D_{0, s}$. When two heteroclinic connections between the two saddle points exist, the two free connecting orbits of each saddle point form either a homoclinic saddle connection or a pair of ss-saddle connections to the 1-source-sink point owing to (ii). When both of the two saddle points have homoclinic saddle connections, we obtain the $h$-unstable pattern with a pair of heteroclinic connections generated by the operation $E_{0}$. On the other hand, when one of the saddle points has a pair of ss-saddle connections and the other has a homoclinic connection, the $h$-unstable pattern is equivalent to the one created by the operation $E_{0, s}$. It is impossible to consider the case when both saddle points have ss-saddle connections simultaneously. Suppose that the two saddle points are connected by three heteroclinic connections, then each saddle point has one free connecting 
orbit. When the free connecting orbits of the two saddle points lead to the 1-source-sink point, we have the $h$-unstable stable pattern $\Phi_{0, s}$. On the other hand, connecting the free connecting orbits heteroclinically yields the $h$-unstable pattern $\Phi_{0}$ with the four heteroclinic connections.

Second, we consider heteroclinic s- $\partial$-saddle connections between a saddle point with $\partial$-saddles on a circular boundary. When there is one heteroclinic connection between them, the two free connecting orbits of the saddle point form a homoclinic saddle connection and the one free connecting orbit should lead to the 1 -source-sink point. Then we have the $h$-unstable streamline pattern generated by the operation $D_{0, s}$. If two of the four free connecting orbits of the saddle point lead to two $\partial$-saddles on the same circular boundary, the other two are either connected to the 1-source-sink point or joined homoclinically. This yields the $h$-unstable streamline pattern created by $E_{2, s}$ in the former case, whilst we have the $h$-unstable pattern generated by the operations $E_{2}$ and $D_{0}$ in the latter case. With three heteroclinic connections between a saddle point and $\partial$-saddles on a circular boundary, we have one free connecting orbit of the saddle point and one free $\partial$-saddle on the circular boundary, whose existence is assured by (ii) and (iii). If the free connecting orbit leads to the free $\partial$-saddle, we have the $h$-unstable pattern $\Psi(2)$. On the other hand, the $h$-unstable pattern $\Psi_{s}(2)$ is obtained when the free connecting orbit and the free $\partial$-saddle connection lead to the 1-source-sink point.

Third, we deal with heteroclinic $\partial$-orbits between $\partial$-saddles on different two circular boundaries. In view of (iii), an arbitrary positive number of heteroclinic $\partial$-orbits can exist on a circular boundary. Suppose that there exists one heteroclinic $\partial$-orbit between the two circular boundaries, then each boundary must have at least one free $\partial$-saddle owing to (iii). When both of the free $\partial$ saddles lead to the 1-source-sink point, the $h$-unstable streamline pattern $D_{2, s}$ is obtained. On the other hand, joining them together, we have the $h$-unstable pattern generated by the operation $D_{2}$ with a pair of heteroclinic $\partial$-orbits. Generally, with $2 n-1(n \geq 2)$ heteroclinic $\partial$-orbits between two circular boundaries, we have the $h$-unstable streamline pattern generated by the operation $\Xi_{s}(2 n-1)$ when the free $\partial$-saddles on the two boundaries are connected to the 1-source-sink point, and the $h$-unstable streamline pattern generated by the operation $\Xi(2 n-1)$ when they are joined heteroclinically. Consequently, we form the following proposition.

Proposition 4.1. H-unstable streamline patterns with a heteroclinic pair, whose small perturbation in $\mathcal{H}^{r}$ or $\chi_{1}^{r}$ give rise to structurally stable streamline patterns, are created by the fourteen operations introduced in $\S \S 4.1$ and 4.2.

Table 1 is the list of all possible transitions between two structurally stable Hamiltonian vector fields through $h$-unstable vector fields. The transition between two structurally stable vector fields could occur when their maximal words $W_{1}$ and $W_{2}$ contain the sequences $S_{1}$ and $S_{2}$ as their subwords and the difference of their indices is equivalent to the vector $\boldsymbol{d} \in \mathbb{Z}^{5}$ shown in the table.

\section{Streamline patterns with a pinching point}

\subsection{Operations creating a pinching point}

A pinching point is obtained by merging two $\partial$-saddles on the same circular boundary together. In what follows, we introduce operations that construct $p$-unstable streamline patterns in $\mathcal{D}_{\zeta}(M)$ from structurally stable patterns in $\mathcal{D}_{\zeta}(M-1)$.

(Fundamental pattern $\Lambda$ ) According to [15], there is no structurally stable streamline pattern in $\mathcal{D}_{\zeta}(0)$ except the fundamental patterns $I$ and $I I$ with the 1 -source-sink point. However, if 
the existence of a pinching point is permitted, we can obtain a $p$-unstable pattern with the 1source-sink point in $\mathcal{D}_{\zeta}(0)$, say Pattern $\Lambda$, whose ss-saddle connection diagram is illustrated in Figure 21(a). Perturbing the pattern $\Lambda$ in $\chi_{1}^{r}$, we have a transition between the fundamental patterns $I$ and $I I$, which gives rise to the change of index, $\operatorname{ind}_{I}(I)-\operatorname{ind}_{I I}(I I)=(-1,1,0,0,0)$. On the other hand, when the homoclinic pinching orbit in the pattern $\Lambda$ is enclosed by a $\partial$-saddle connection as in Figure 21(b), we have the structurally stable streamline patterns $I C$ and $I I B_{2}$ by perturbation in $\chi_{1}^{r}$. The index then changes as $\operatorname{ind}_{I}(I C)-\operatorname{ind}_{I I}\left(I I B_{2}\right)=(-1,1,0,-1,1)$. One can then introduce new word representations of $p$-unstable patterns with a pinching point starting from the fundamental patten $\Lambda$ followed by $A_{0}, A_{2}, B_{0}, B_{2}$ and $C$, which we refer to as maximal $\Lambda$-words. The perturbation of a $p$-unstable pattern represented by a maximal $\Lambda$-word always brings us streamline patterns with the maximal $I$-word and $I I$-word followed by the same sequence of operations except in the following special case. If the sequence of a $\Lambda$-word contains the operation $A_{2}$, one of its perturbed patterns represented by the $I I$-word is converted into a $I$-word owing to the equivalence relation $I I A_{2}=I A_{0}$. For example, the perturbed structurally stable patterns of $\Lambda A_{0} A_{2} B_{0} C$ are represented by $I A_{0} A_{2} B_{0} C$ and $I I A_{0} A_{2} B_{0} C=I A_{0} A_{0} B_{0} C$.

(Operation $\Delta_{1}$ ) By $\Delta_{1}$, we symbolize the operation attaching a homoclinic pinching orbit to a circular boundary in structurally stable streamline patterns as shown in Figure 22(a). The operation increases the genus element by one, and one 1- $\partial$-saddle to the vector field.

The operation is applied to any physical boundary without $\partial$-saddle as in Figure 23(a) and its perturbed patterns are represented by $B_{0}$ and $B_{2}$. This induces the change of index $\operatorname{ind}_{*}\left(B_{0}\right)-$ $\operatorname{ind}_{*}\left(B_{2}\right)=(0,0,1,-1,0)$ for $*=I, I I$ or $O$. Applying $\Delta_{1}$ to the fundamental patterns $I$ and $I I$, we obtain the $p$-unstable streamline patterns represented by $I \Delta_{1}$ in Figure 23(b) and $I I \Delta_{1}$ in Figure 23(c). Perturbation of these $p$-unstable patterns yields $I A_{0}$ and $I C$ for $I \Delta_{1}$, and $I I B_{0}$ and $I I B_{2}$, which is equivalent to the pattern $I I C$, for $I I \Delta_{1}$. Their corresponding index changes are given respectively by:

$$
\operatorname{ind}_{I}\left(I A_{0}\right)-\operatorname{ind}_{I}(I C)=(1,0,0,0,-1), \quad \operatorname{ind}_{I I}\left(I I B_{0}\right)-\operatorname{ind}_{I I}\left(I I B_{2}\right)=(0,0,1,-1,0) .
$$

Next, the application of $\Delta_{1}$ to the structurally stable orbits generated by the operations $A_{0}$ and $B_{0}$ gives rise to the $p$-unstable patterns $A_{0} \Delta_{1}$ and $B_{0} \Delta_{1}$ shown in Figures $23(\mathrm{~d})$ and (e). But it is unnecessary to consider these patterns, since they are just obtained by applying $\Delta_{1}$ to a physical boundary enclosed by a homoclinic connection.

Finally, Figure 24 shows all $p$-unstable patterns obtained by applying $\Delta_{1}$ to the structurally stable patterns created by the operations $A_{2}, B_{2}$ and $C$. It is unnecessary to consider the $p$ unstable pattern $A_{2} \Delta_{1}$, since it is topologically equivalent to $I \Delta_{1}$ as illustrated in Figure 24(a). In the pattern $B_{2}$, there are two circular boundaries to which a homoclinic pinching point is attached. However, it is sufficient to consider the case when we append a homoclinic pinching orbit to the physical boundary with $\partial$-saddles, since appending a homoclinic orbit to the other circular boundary without a $\partial$-saddle yields the same transition as $\Delta_{1} B_{2}$ shown in Figure 23(a). Consequently, the $p$-unstable pattern $B_{2} \Delta_{1}$ in Figure 24(b) is a transient state between the two structurally stable patterns $B_{0} B_{2}$ (or $B_{2} B_{0}$ ) and $B_{2} C$ inducing the following change of index.

$$
\begin{aligned}
\operatorname{ind}_{O}\left(B_{2} B_{0}\right)-\operatorname{ind}_{O}\left(B_{2} C\right) & =\operatorname{ind}_{I}\left(B_{2} B_{0}\right)-\operatorname{ind}_{I}\left(B_{2} C\right) \\
& =\operatorname{ind}_{I I}\left(B_{2} B_{0}\right)-\operatorname{ind}_{I I}\left(B_{2} C\right)=(0,0,1,0,-1) .
\end{aligned}
$$

We have different $p$-unstable streamline patterns when applying the operation $\Delta_{1}$ to a circular boundary with a $\partial$-saddle connection generated by the operation $C$. If the circular boundary 
has a pair of ss- $\partial$-saddle connections, we obtain the pattern represented by $A_{2} C \Delta_{1}$, which is equivalent to $I C \Delta_{1}$ as shown in Figure 24(c). The perturbation of the pattern in $\chi_{1}^{r}$ yields the streamline patterns $I C B_{0}$ and $I C C$, whose change of index is given by $\operatorname{ind}_{I}\left(I C B_{0}\right)-\operatorname{ind}_{I}(I C C)=$ $(0,0,1,0,-1)$. Applying $\Delta_{1}$ to the circular boundary without the ss- $\partial$-saddle connection, the $p$ unstable pattern is represented by $B_{2} C \Delta_{1}$ shown in Figure $24(\mathrm{~d})$, which is equivalent to $B_{2} \Delta_{1} C$.

(Operations $M_{1, s}, M_{1, i}$ and $M_{1, o}$ ) When a $\partial$-saddle connection is added to a boundary so that it shares an endpoint with the ss- $\partial$-saddle connection on the same boundary as shown in Figure 22(b), the operation is denoted by $M_{1, s}$. The operation increases one $\partial$-saddle and the genus element by one to the vector field. An ss- $\partial$-saddle connection exists either in the fundamental pattern $I$ or in the structurally stable pattern created by the operation $A_{2}$. The perturbation of the $p$-unstable pattern $I M_{1, s}$ in $\chi_{1}^{r}$ yields the two structurally stable patterns $I A_{0}$ and $I C$. See Figure 25(a). Since $A_{2} M_{1, s}$ is equivalent to $I M_{1, s}$ as illustrated in Figure 25(b), it suffices to consider the $p$-unstable pattern $I M_{1, s}$.

On the other hand, $M_{1, i}$ and $M_{1, o}$ symbolize the operations adding a $\partial$-saddle connection to a boundary that shares a $\partial$-saddle of another $\partial$-saddle connection on the same boundary. When the new $\partial$-saddle connection is added to the exterior of the existing $\partial$-saddle connection, we refer to the operation as $M_{1, o}$, while it is called $M_{1, i}$ if the new $\partial$-saddle is placed inside the $\partial$-saddle connection. See the illustrations in Figure 22(c). Remembering that $\partial$-saddle connections in the structurally stable patterns are created by the operations $B_{2}$ and $C$, we consider the four $p$-unstable patterns represented by $B_{2} M_{1, o}, B_{2} M_{1, i}, C M_{1, o}$ and $C M_{1, i}$.

Figure 26(a) shows the $p$-unstable patterns $B_{2} M_{1, o}$ and $B_{2} M_{1, i}$. Their perturbed structurally stable patterns are represented by the same words $B_{0} B_{2}$ (or $B_{2} B_{0}$ ) and $B_{2} C$, although their corresponding streamline patterns are different. We then have the following change of index:

$$
\begin{aligned}
\operatorname{ind}_{O}\left(B_{0} B_{2}\right)-\operatorname{ind}_{O}\left(B_{2} C\right) & =\operatorname{ind}_{I}\left(B_{0} B_{2}\right)-\operatorname{ind}_{I}\left(B_{2} C\right) \\
& =\operatorname{ind}_{I I}\left(B_{0} B_{2}\right)-\operatorname{ind}_{I I}\left(B_{2} C\right)=(0,0,1,0,-1) .
\end{aligned}
$$

The application of $M_{1, o}$ and $M_{1, i}$ to a $\partial$-saddle connection created by the operation $C$ gives rise to four different $p$-unstable patterns, which are considered separately as follows. When the circular boundary with the $\partial$-saddle connection has a pair of ss- $\partial$-saddle connections, we obtain the $p$-unstable patterns $I C M_{1, o}$ and $I C M_{1, i}$, which are shown in Figure 26(b). Under a perturbation in $\chi_{1}^{r}$, the $p$-unstable pattern $I C M_{1, o}$ reduces to the stable streamline patterns $I C B_{0}$ and $I C C$, while $I C M_{1, i}$ is modified to $I A_{0} C$ or $I C C$. Accordingly, we have the change of index, $\operatorname{ind}_{I}\left(I C B_{0}\right)-\operatorname{ind}_{I}(I C C)=(0,0,1,0,-1)$ for $I C M_{1, o}$ and $\operatorname{ind}_{I}\left(I A_{0} C\right)-\operatorname{ind}_{I}(I C C)=$ $(1,0,0,0,-1)$ for $I C M_{1, i}$. When the $p$-unstable structure is enclosed by a $\partial$-saddle connection, the pattern $I C C M_{1, i}$ in Figure 26(c) gives rise to another transition between the two structurally stable pattern represented by $I C C B_{0}$ (or $I C B_{0} C$ ) and $I C C C$, where corresponding change of index is given by $\operatorname{ind}_{I}\left(I C C B_{0}\right)-\operatorname{ind}_{I}(I C C C)=(0,0,1,0,-1)$. The $p$-unstable pattern $I C C M_{1, o}$ is the same as $I C M_{1, o} C$. On the other hand, the two $p$-unstable pattens $B_{2} C M_{1, o}$ and $B_{2} C M_{1, i}$ arise by applying the operations to a $\partial$-saddle connection attached to a circular boundary without ss- $\partial$-saddle connections. However, these patterns are equivalent to $B_{2} M_{1, o} C$ and $B_{2} M_{1, i} C$.

\section{$5.2 \quad P$-unstable streamline topologies with a pinching point}

Let us again recall that any $\partial$-saddle on a physical boundary in structurally stable patterns leads either to the 1 -source-sink point or to another $\partial$-saddle on the same boundary. First, when two $\partial$-saddles on the same circular boundary are both connected to the 1-source-sink point, we 
obtain the fundamental pattern $\Lambda$ by joining them together. Next, when a $\partial$-saddle on a boundary is connected to the 1 -source-sink point and the other $\partial$-saddle is an endpoint of a different $\partial$-saddle on the same boundary, merging the two $\partial$-saddles yields the $p$-unstable streamline pattern generated by the operation $M_{1, s}$. Finally, the $p$-unstable streamline pattern $\Delta_{1}$ appears by merging two $\partial$-saddles connected by a $\partial$-saddle connection. On the other hand, we obtain the $p$ unstable streamline patterns created by $M_{1, i}$ and $M_{1, o}$ when $\partial$-saddles connected by two different $\partial$-connections are joined together. Consequently, we form the following proposition.

Proposition 5.1. P-unstable streamline patterns with a pinching point, whose small perturbation in $\mathcal{H}^{r}$ or $\chi_{1}^{r}$ gives rise to two structurally stable streamline patterns, are constructed from the fundamental pattern $\Lambda$ or created by the five operations given in $\S 5.1$.

All possible transitions through $p$-unstable Hamiltonian vector fields are listed in Table 2.

\section{Transitions between structurally stable Hamiltonian vector fields}

Using Table 1 and 2, it is possible to determine transient structurally unstable streamline patterns between structurally stable Hamiltonian vector fields. Let us first note that we are unable to obtain any transition between streamline patterns represented by $O$-words and those by $I$-words and $I I$-words owing to the existence/non-existence of the 1 -source-sink point. Suppose that two structurally stable vector fields with the same number of genus elements are given. Then we have the following proposition.

Proposition 6.1. Let $W_{1}$ and $W_{2}$ be the word representations of two structurally stable Hamiltonian vector fields with the same number of genus elements. Then the existence of a triplet $\left(S_{1}, S_{2}, \boldsymbol{d}\right)$ in Table 1 and 2 such that $S_{1} \subseteq W_{1}$ and $S_{2} \subseteq W_{2}$ and $\operatorname{ind}_{*}\left(W_{1}\right)-i n d_{*}\left(W_{2}\right)=\boldsymbol{d}$ for a certain $* \in\{O, I, I I\}$ is required to result in a transition between these structurally stable vector fields.

The existence of triplet $\left(S_{1}, S_{2}, \boldsymbol{d}\right)$ is a necessary condition to identify possible transitions. For instance, when we consider the two streamline patterns represented by the maximal $I$-words, $W_{1}=I A_{0} A_{2} C B_{0}$ and $W_{2}=I A_{0} C B_{0} B_{2}$, we have $\operatorname{ind}_{I}\left(W_{1}\right)-\operatorname{ind}_{I}\left(W_{2}\right)=(0,1,0,-1,0)$ and $I A_{2} C \subseteq W_{1}, I C B_{2} \subseteq W_{2}$. Then, we can find the triplet $\left(I A_{2} C, I C B_{2},(0,1,0,-1,0)\right)$ in Table 1 , which indicates the transition could occur through an $h$-unstable pattern with the local streamline structure of $X=I C D_{2} \approx A_{2} C D_{2}$ as shown in in Figure 15(b). On the other hand, we find the other triplet $\left(I A_{0} A_{2}, I A_{0} B_{2},(0,1,0,-1,0)\right)$ with $I A_{0} A_{2} \subseteq W_{1}$ and $I A_{0} B_{2} \subseteq W_{2}$, which suggests another transition through an $h$-unstable pattern containing the local streamline structure of $X=$ $I A_{0} E_{2, s}$. We must also note that it is impossible to determine which transition really occurs just from the maximal words. Hence, we not only show the existence of triplet for given maximal words, but we also need to compare the structurally stable streamlines obtained by the perturbation of the transient pattern with the given patterns. If these streamline topologies coincide, we can conclude the existence of the transition through the marginal pattern that contains the structure $X$ corresponding to the triplet and thus the symbol $X$ is available as a label of the transition.

Now we have the word representations of all structurally stable streamline topologies and all possible transitions between them owing to Proposition 6.1, it is easy to visualize the global network of transitions as a graph, which we call a transition graph, provided that the number of genus elements is fixed. Figure 27 shows the transition graph of structurally stable Hamiltonian vector fields with the 1 -source-sink point on $\mathcal{D}_{\zeta}(1)$, in which the ss-saddle connection diagrams with the maximal words are drawn as nodes and two nodes are connected by an edge if there exists 
a structurally unstable Hamiltonian vector field between them. The label of each edge symbolizes what kind of transition occurs between these nodes. Figure 28 shows the transition graph of the structurally stable Hamiltonian vector fields on $\mathcal{D}_{\zeta}(2)$, which indicates that the transitions of streamline topologies can be extremely complicated even for the small number of genus elements.

\section{Applications to incompressible fluid flows}

Proposition 6.1 is utilized to determine transitions between streamline patterns of incompressible fluid flows, since they induce Hamiltonian vector fields. Figure 29 shows streamline patterns of a potential flow around a unit circle in the presence of a uniform flow. This is one of the fundamental flows, whose complex potential is given by

$$
W(\zeta)=U\left(\zeta+\frac{1}{\zeta}\right)+\frac{\Gamma}{2 \pi i} \log \zeta,
$$

where $U$ is the flux of the uniform flow and $\Gamma$ denotes the circulation around the circle. As $\Gamma$ changes from $-2.5 \pi$ to $-5.5 \pi$ for fixed $U=1$, we observe a transition of streamline topologies as shown in Figure 29(a) and Figure 29(b) whose maximal words are represented by $I$ and $I I$ respectively. Since $\operatorname{ind}_{I}(I)-\operatorname{ind}_{I I}(I I)=(-1,1,0,0,0)$, it follows from Table 2 that there exists the transient $p$-unstable pattern $X=\Lambda$ between these streamline patterns. This is certainly confirmed by the streamline pattern for $U=1$ and $\Gamma=-4 \pi$ shown in Figure 29(c).

In what follows, we show two examples of incompressible and viscous flows to observe some features of the present method. Since the theory of word representation is developed for Hamiltonian vector fields with the slip boundary condition, it is not, in principle, applicable to viscous flows that satisfy the no-slip boundary condition. Nevertheless, the theory is still applicable under a certain circumstance where the boundary layer is sufficiently thin compared to the scale of the whole flow domain or it is less developed so that the flow can be approximated by an inviscid and incompressible flow with the slip-boundary condition along the outer surface of the layer. Here, isolated vortex structures shed from the boundary owing to the viscous effect can be described as genus elements. In the following two examples, we assume that the situation is valid.

We first consider a flow behind a circular boundary in order to clarify the difference between a preceding bifurcation analysis and the present combinatorial method. Brøns et al.[7] have revealed some scenarios of topological streamline changes of this flow by a bifurcation analysis as shown in Figure 30. For the Reynolds number Re $\sim 45$, the streamline pattern changes periodically between the mirror symmetric patterns with the same word $I A_{0} C$ as in Figure 30(a), which is induced by a Hopf bifurcation. Although we are unable to distinguish these mirror symmetric patterns with their word representations, the present method can identify the transition between them. As a matter of fact, it follows from $\boldsymbol{d}=\operatorname{ind}_{I}\left(I A_{0} C\right)-\operatorname{ind}_{I}\left(I A_{0} C\right)=\mathbf{0}, S_{1}=S_{2}=I A_{0} C$ and Table 1 that the marginal state is represented by the $h$-unstable pattern $I \Psi_{s}(2)$ as shown in Figure 18(a). Let us note that the present approach conclude nothing about the Hopf bifurcation, since we just consider the transition between them up to topological equivalence.

For $\operatorname{Re} \sim 100$ up to around 200, the transition of streamline topologies through a streamline pattern with a cusp singularity as shown in Figure 30(b) is generically observed[12]. This transition is induced by the saddle-center bifurcation where a cusp singularity appears in a streamline[7]. It is important to notice that the combinatorial approach presented in this paper is unable to deal with the saddle-center bifurcation in principle. This is because a cusp singularity is indistinguishable with a degenerate 0 -saddle up to topological equivalence, whose existence is excluded as discussed in $\S 3$. On the other hand, combined with the bifurcation analysis, we could extend the present 
method so that it can deal with the saddle-center bifurcation in a sense. That is to say, since the two structurally stable streamline patterns in Figure 30(b) are represented by $I A_{0} C$ and $I C$ respectively, the transition between them is described as the increase (or the decrease) of the word length that corresponds to the operation adding (or removing) a homoclinic saddle point to (or from) an ss-orbit. In consideration of the fact that the saddle-center bifurcation is a generic mechanism of the transition[7], we could conclude that the transition between the patterns $I C$ and $I A_{0} C$ occurs generically through the saddle-center bifurcation.

The last example deals with an evolution of a $2 \mathrm{D}$ incompressible and viscous flow around a single flat plate in the presence of a uniform flow considered in [13]. The evolution was numerically computed using the volume penalization method[2]. Figure 31 shows the snapshots of the vorticity evolution in the left column and the instantaneous streamline patterns at the same moment in the right column extracted from the stream function, to which we assign the maximal words. In the illustrations of the streamline topologies, the rotational direction of the flow is symbolized by + for the counter-clockwise rotation and by - for the clockwise rotation. In [13], a relation between vortex structures around the flat plate and the lift-to-drag ratio acting on the plate has also been discussed. That is to say, the lift to drag ratio becomes maximum at $t=5.5$ owing to the entrapped vortex structures above the flat plate in Figure 31(a). The entrapped vortex structure has broken at $t=6.6$ as we observe in Figure 31(b). The lift-to-drag ratio then becomes the minimum at $t=7.7$ when the vortex structures are isolated and going away from the plate as in Figure 31(c). We now describe how the entrapped vortex structure disappears from the topological point of view using Proposition 6.1.

The streamline pattern at $t=5.5$ in Figure $31(\mathrm{a})$ is represented by $W_{0}=I C C B_{0}$, in which a vortex structure corresponding to the subword $C B_{0}$ is entrapped by a $\partial$-saddle connection above the plate. The streamline pattern transfers to the one represented by $W_{1}=I A_{0} C B_{0}$ at $t=6.6$ as shown in Figure 31(b). Since $\operatorname{ind}_{I}\left(W_{1}\right)-\operatorname{ind}_{I}\left(W_{0}\right)=(1,0,0,0,-1), I A_{0} C \subseteq W_{1}$ and $I C C \subseteq W_{0}$, we can find the triplet $\left(I A_{0} A_{0}, I C C,(1,0,0,0,-1)\right)$ in Table 2 showing that the possible transition is either $X=I M_{1, s}, I \Delta_{1}$ or $I C M_{1, i}$. Moreover, comparing the two streamlines of Figure 31(a) and (b), we conclude that the transition through $X=I M_{1, s}$ occurs. The transient $p$-unstable pattern between the streamline patterns at $t=5.5$ and 6.6 is shown in Figure 32(a). The word representation of the streamline pattern at $t=7.7$ in Figure $31(\mathrm{c})$ is $W_{2}=I A_{0} A_{0} C$. It follows from $\operatorname{ind}_{I}\left(W_{2}\right)-\operatorname{ind}_{I}\left(W_{1}\right)=(1,0,-1,0,0)$ that the possible transition is either $X=I C D_{0}, A_{0} E_{0, s}$ or $I C \Psi(2)$. The transition via $X=I C \Psi(2)$ is impossible because $I C C B_{0} \nsubseteq W_{1}$ and $I A_{0} C C \nsubseteq W_{2}$. We can also exclude the transition via $X=I C D_{0}$ by comparing the perturbed patterns with the two streamlines in Figures 31(b) and (c). Consequently, we conclude that the transition between the two streamline patterns $W_{1}=I A_{0} C B_{0}$ and $W_{2}=I A_{0} A_{0} C$ occurs thorough the $h$-unstable streamline pattern with the local streamline structure of $X=A_{0} E_{0, s}$ as shown in Figure 32(b).

The streamline pattern at $t=8.8$ is represented by $I A_{0} A_{0} C C$, in which there appears a new vortex structure from the trailing edge of the plate, which is created by the off-wall vortex creation according to the bifurcation analysis[10]. Since the vortex creation is regarded as the bifurcation through a degenerate 0 - $\partial$-saddle topologically, the present method can not deal with this transition as we have observed in the previous example. On the other hand, the change of the streamline pattern from $t=7.7$ to 8.8 is described as appending a word $C$ to the word representation. Hence, combined with the result of the bifurcation analysis[10], we could say this change is generically brought by the off-wall vortex creation through a degenerate 0 - $\partial$-saddle.

After $t=9.0$, we sample the instantaneous streamline patterns every 0.4 time step and confirm that the maximal word representation is unchanged until $t=11.4$. At $t=11.8$, the streamline pattern changes largely as shown in Figure 31(e), whose maximal word is given by $W_{4}=I A_{0} C C B_{0}$. 
It indicates that another transition occurs between $t=11.4$ and 11.8. Checking the existence of the triplet $\left(S_{1}, S_{2}, \boldsymbol{d}\right)$ for $\boldsymbol{d}=\operatorname{ind}_{I}\left(W_{3}\right)-\operatorname{ind}_{I}\left(W_{4}\right)=(1,0,-1,0,0)$ in Table 1, we find that the transitions through the marginal states $X=I C \Psi(2), A_{0} E_{0, s}$ and $I C D_{0}$ are possible. If there occurs the transition via $X=I C \Psi(2)$, whose transient streamline pattern is illustrated in Figure 33(a), it transfers to the structurally stable streamline pattern in Figure 33(b). However, the rotational directions of the figure eight pattern represented by $B_{0}$ are opposite from those contained in the streamline pattern at $t=11.8$ in Figure 31(e). Consequently, the transition via $X=I C \Psi(2)$ is impossible. On the other hand, when there occur the transitions via $X=A_{0} E_{0, s}$ and $X=I C D_{0}$ in Figure 33(c) and (e), they give rise to the structurally stable patterns as shown in Figure 33(d) and (f), which are different from that in Figure 31(e). This observation indicates that the structurally stable pattern at $t=11.4$ can not transfer to that at $t=11.8$ directly. Hence, there exist some intermediate transitions before reaching the pattern at $t=11.8$.

In order to see the real route in the evolution, we need to sample the instantaneous snapshots of the evolution with a finer time step between $t=11.4$ and 11.8. On the other hand, we could use the transition graph as in $\S 6$ for $M=4$ to discuss the shortest route from the streamline pattern $I A_{0} A_{0} C C$ in Figure $31(\mathrm{~d})$ to that with $I A_{0} C C B_{0}$ in Figure $31(\mathrm{e})$, which is given in Figure 34. The route is realized via the transitions $X=I M_{1, s}$ and $I C M_{1, i}$. Figure 34(a) shows the transient state that leads to the structurally stable streamline pattern represented by $I C C C B_{0}$ in Figure 34(b), where all vortex structures are entrapped by a $\partial$-saddle connection above the flat plate. Then the endpoints of the different $\partial$-saddle connections join, which gives rise to the marginal $p$-unstable streamline pattern with $X=I C M_{1, i}$ as illustrated in Figure 34(c). We finally obtain the structurally stable pattern represented by $W_{4}=I A_{0} C C B_{0}$ as desired. We must note that this is just a conjecture among many possible routes of transitions.

\section{Summary and discussion}

This paper has presented a combinatorial procedure to provide a list of possible transitions between two structurally stable Hamiltonian vector fields with the slip boundary condition in multiply connected domains from their maximal word representations. The transient pattern is effectively identified up to topological equivalence under the assumptions that the number of genus elements is fixed and that of stagnation points is finite. In this case, any transient streamline pattern between them is generically represented by either an $h$-unstable pattern with a heteroclinic pair or a $p$-unstable pattern with a pinching point, since $h$-unstable and $p$-unstable Hamiltonian vector fields are open dense in the set of structurally unstable Hamiltonian vector fields satisfying these assumptions. The derivation of possible transitions proceeds as follows. First, for maximal word representations $W_{1}$ and $W_{2}$ assigned to two streamline patterns, we compute the difference of their indices $\boldsymbol{d}=\operatorname{ind}_{*}\left(W_{1}\right)-\operatorname{ind}_{*}\left(W_{2}\right)$ for an appropriate $* \in\{I, I I, O\}$. Next, we look for transitions $X$ in Tables 1 and 2 with the difference $\boldsymbol{d}$. Finally, we confirm if the subwords $S_{1}$ and $S_{2}$ corresponding to $X$ are contained in $W_{1}$ and $W_{2}$, i.e. $S_{1} \subseteq W_{1}$ and $S_{2} \subseteq W_{2}$. The existence of triplet $\left(S_{1}, S_{2}, \boldsymbol{d}\right)$ for $W_{1}$ and $W_{2}$ shows a transient streamline pattern containing the local streamline structure symbolized by $X$ between the streamline patterns represented by $W_{1}$ and $W_{2}$. On the other hand, since it is only a necessary condition, we need to perturb the transient pattern and check whether or not the perturbed streamline patterns are topologically equivalent to the given two structurally stable patterns. This procedure is applicable to not only transitions of streamline topologies of incompressible and inviscid flows but also those of viscous flows under the assumption that the boundary layer is sufficiently thin compared to the scale of the whole flow or it is less developed and almost laminar. 
We discuss the difference between the combinatorial approach and the bifurcation analysis for flow problems in the preceding research[7, 8, 10]. These bifurcation analysis have revealed that the saddle-center bifurcation and the off-wall vortex creation are generic mechanisms that give rise to the changes of streamline topologies in many fluid flows. On the other hand, it is impossible to deal with these changes with the combinatorial approach in principle, since these bifurcations occur through degenerate $0-(\partial-)$ saddles whose existence is prohibited in the present paper. The non-coverage of these generic bifurcations via a degenerate $0-(\partial$-) saddle is a drawback of the present method. However, we must note that these bifurcations give rise to the generation (or disappearance) of an elliptic fixed point, which results in the increase (or decrease) of $A_{0}, B_{0}$ or $C$ in the word representation. Hence, when we allow the change of streamline topologies with appending (or removing) $A_{0}, B_{0}$ or $C$ to (or from) its word representation, we could expect that this change has been brought by the saddle-center bifurcation and the off-wall vortex creation in a generic manner thanks to the results of the preceding bifurcation analysis. Even if such an extension is possible, we hardly conclude that the marginal state between these two streamline topologies is a pattern with a cusp singularity within topological equivalence.

In spite of this drawback, the present combinatorial approach has some merits in the analysis of streamline topologies. First, we have theoretically shown that the transition of streamline topologies with the same number of genus elements occurs generically through either of an $h$ unstable or a $p$-unstable Hamiltonian vector field. It reveals the existence of new generic and global transitions that are not obtained by the bifurcation analysis such as shown in Figure 12(c), Figure 19 and Figure 20 for instance. Moreover, the transition graphs like Figure 27 and Figure 28 are also new results brought by the combinatorial analysis. With the transition graphs, we can easily identify what kinds of transitions are possible or impossible for a given streamline pattern as demonstrated in $\S 7$. Second, the present method is completely combinatorial and any specific form of Hamiltonian is unnecessary, since we obtain a list of possible transitions between two given streamline topologies only by comparing their maximal words. Therefore, it is applicable to instantaneous snapshots of streamline patterns observed in laboratory experiments and numerical simulations no matter how complex topological structure they may have. This indicates that, with using the combinatorial approach, one can compress a large amount of long-time flow evolution data into a small size of string data with maximal words. Let us remark that, as we see in the last example in $\S 7$, the poor sampling of instantaneous streamline patterns results in an insufficient description.

Let us finally comment on the uniqueness of the word representations. As we have observed in the example of the cylinder wakes in $\S 7$, the mirror symmetric streamline patterns have the same word representation, although the transition between them is identified by the combinatorial method. In the example of the flow around the flat plate in $\S 7$, even if we can obtain all possible transitions of streamline patterns, we need a pattern matching to determine which one among them really occurs. These phenomena are caused by the uniqueness problem as discussed in [13, 15]. That is to say, the word representations are in many-to-one correspondence to structurally stable streamline topologies, since every maximal word is assigned to an equivalence class of structurally stable Hamiltonian vector fields. Hence, we are unable to distinguish each streamline pattern in the same equivalence class. However, this is not a serious problem, since not maximal words but streamline patterns are usually given as a result of numerical simulations and laboratory experiments, to which we can assign the unique maximal word[13]. In addition, we have recently developed a tree representation that is in one-to-one correspondence to each streamline topology in order to resolve this uniqueness problem[14]. This tree representation can compensate for the drawback caused by the lack of uniqueness, which will be reported in near future. 


\section{Acknowledgments}

We thank the anonymous referees for their useful comments and for bringing some important references to our attention.

\section{References}

[1] H. Aref and M. Brøns, On stagnation points and streamline topology in vortex flows, J. Fluid Mech. 370 (1998) pp. 1-27.

[2] P. Angot, C.-H. Bruneau and P. Fabrie, A penalization method to take into account obstacles in incompressible viscous flows, Num. Math. 81 (1999) pp. 497-520.

[3] P. G. Bakker, Bifurcations in flow patterns. Kluwer Acad. Publ., Dordrecht, (1991).

[4] M. Brøns, Topological fluid mechanics of axisymmetric flows. In J. J. Sørensen, E. J. Hopfinger and N. Aubry eds, Simulation and identifications of organized structures in flows, Kluwer, (1999) pp. 213-222.

[5] M. Brøns, L. K. Voigt and J. N. Sørensen, Streamline topology of steady axisymmetric vortex breakdown in a cylinder with co- and counter-rotating end-covers, J. Fluid Mech. 401 (1999) pp. 275-292.

[6] M. Brøns, Streamline topology: Patterns in fluid flows and their bifurcations, Adv. appl. math. 41 (2007) pp. 1-42.

[7] M. Brøns, B. Jakobsen, K. Niss, A. V. Bisgaard and L. K. Voigt, Streamline topology in the near wake of a circular cylinder at moderate Reynolds numbers, J. Fluid Mech. 584 (2007) pp. 23-43. doi:10.1017/S0022112007006234

[8] M. Brøns and J. N. Hartnack, Streamline topologies near simple degenerate critical points in two-dimensional flow away from boundaries, Phys. Fluids 11 (1999) pp. 315-324. doi:10.1063/1.869881

[9] M. Cobo, C. Gutierrez, and J. Llibre, Flows without wandering points on compact connected surfaces Trans. Amer. Math. Soc. 362 (2010), no. 9, pp. 4569-4580.

[10] J. N. Hartnack, Streamline topologies near a fixed wall using normal forms, Acta Mechanica 136 (1999) pp. 55-75.

[11] T. Ma and S. Wang, Geometric theory of incompressible flows with applications to fluid dynamics Mathematical Surveys and Monographs, 119. American Mathematical Society, Providence, RI, 2005. x+234 pp. ISBN: 0-8218-3693-5

[12] A. E. Perry, M. S. Chong and T. T Lim, The vortex-shedding process behind two-dimensional bluff bodies, J. Fluid Mech. 116 (1982) pp 77-90.

[13] T. Sakajo, Y. Sawamura and T. Yokoyama, A unique encoding for streamline topologies of inviscid and incompressible flows in multiply connected domains Fluid Dynamics Research 46 (2014) 031411

[14] T. Sakajo and T. Yokoayama, Tree representation of topological streamline patterns of structurally stable 2D Hamiltonian flows in multiply connected domains, preprint. 
[15] T. Yokoyama and T. Sakajo, Word representation of streamline topology for structurally stable vortex flows in multiply connected domains Proc. Roy. Soc. A 469 (2013) doi: 10.1098/rspa.2012.0558 

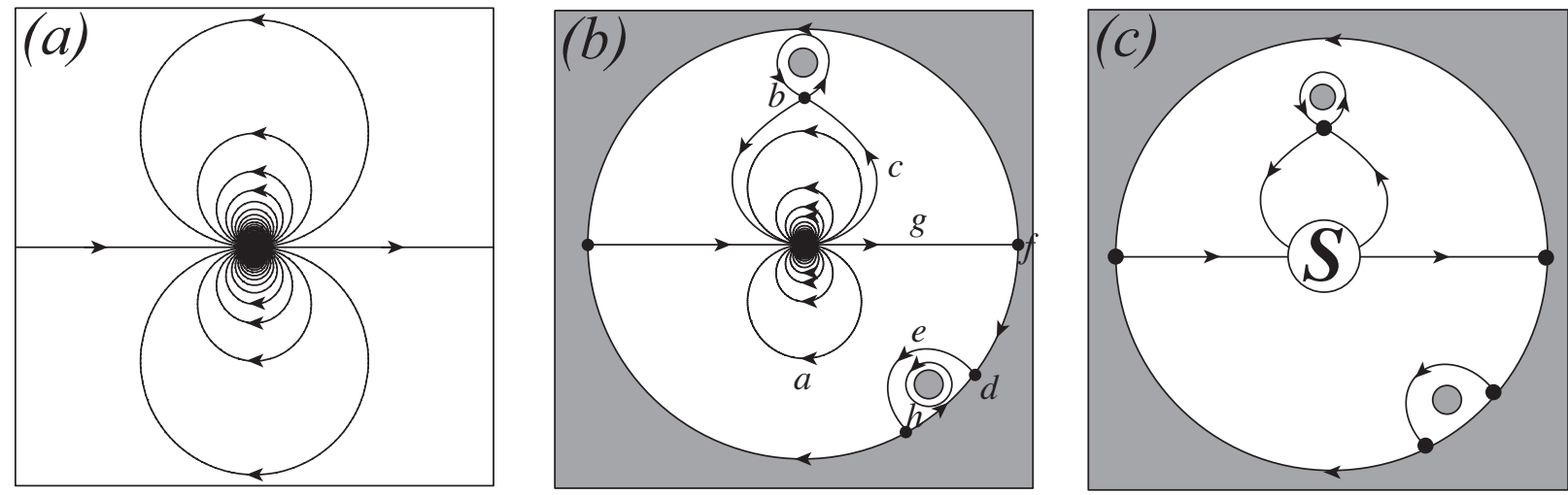

Figure 1: (a) Local streamline structures in the neighborhood of the 1-source-sink point. (b) Streamlines in structurally stable Hamiltonian vector fields. a: ss-orbit, b: homoclinic saddle point, c: ss-saddle connection, d: $\partial$-saddle, e: $\partial$-saddle connection, f: ss- $\partial$-saddle, g: ss- $\partial$-saddle connection, h: periodic orbit. (c) The ss-saddle connection diagram for the streamline pattern in Figure 1(b).

(a)

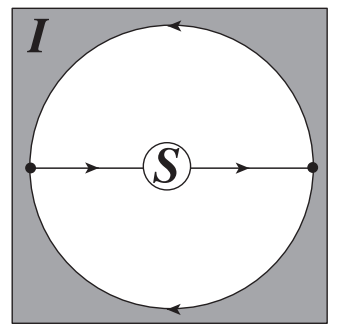

(b)

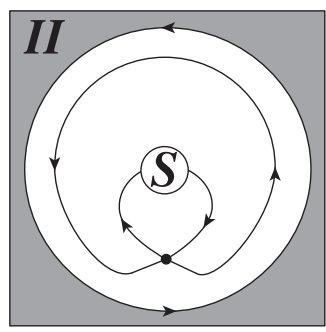

(c)

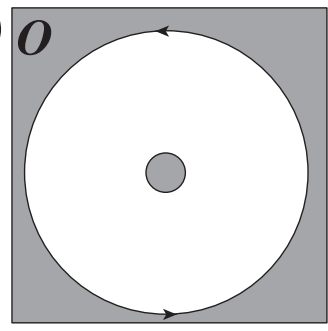

Figure 2: The ss-saddle connection diagrams for the fundamental structurally stable streamline patterns in $\mathcal{D}_{\zeta}(0)$ and $\mathcal{D}_{\zeta}(1)$. (a) Pattern $I$, (b) Pattern $I I$ and (c) Pattern $O$.

(a)

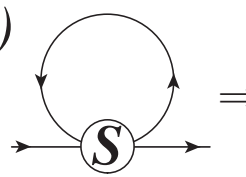

(b)

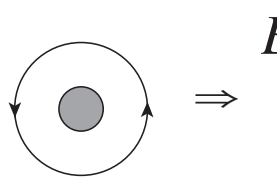

(c)

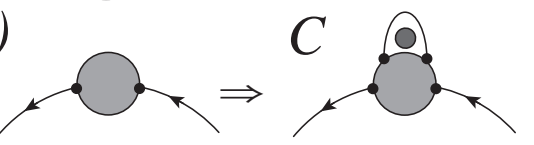

$A_{0}$
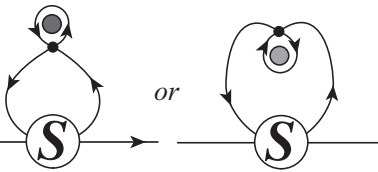

$B_{0}$

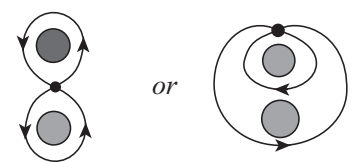

$B_{2}$
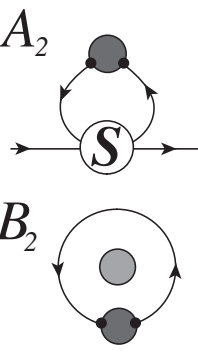

Figure 3: Five operations $A_{0}, A_{2}, B_{0}, B_{2}$ and $C$ that increase the genus element by one, illustrated as a dark gray circular boundary, to the flow domain. Applying these operations to the fundamental structurally stable streamline patterns iteratively, we obtain structurally stable streamline patterns in $\mathcal{D}_{\zeta}(M)$ for any $M$. 
(a)

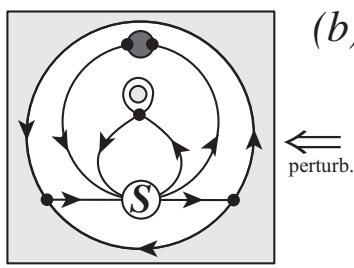

$I A_{0} A_{2}$ (b)

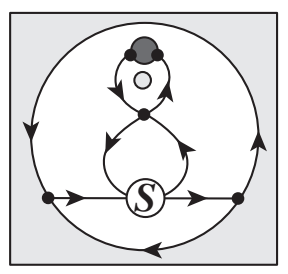

(c)
$\underset{\text { perturb. }}{\Rightarrow}$

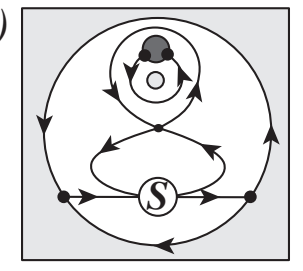

$I A_{0} B_{2}$

Figure 4: An example of a transition between two structurally stable streamline patterns represented by $I A_{0} A_{2}$ and $I A_{0} B_{2}$ through a structurally unstable streamline pattern.
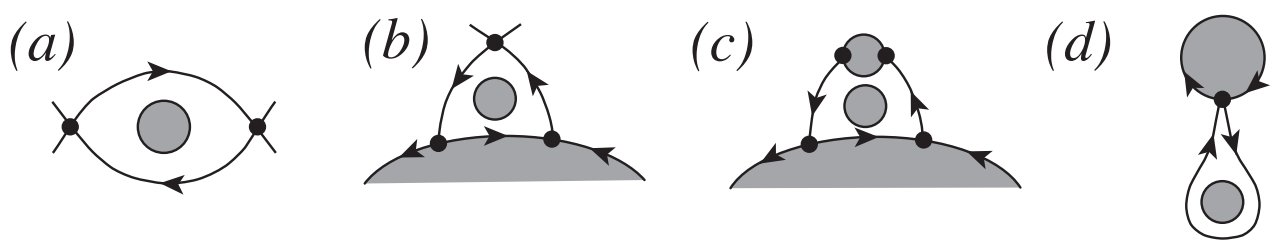

Figure 5: Structurally unstable orbits. (a) A pair of heteroclinic orbits between two saddle points. (b) A pair of s- $\partial$-saddle connections between a saddle point and two $\partial$-saddles. (c) A pair of heteroclinic $\partial$-orbits between $\partial$-saddles. (d) A homoclinic pinching orbit attached at a pinching point on a circular boundary.

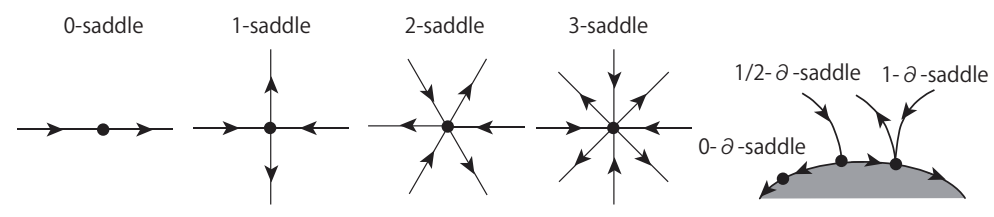

Figure 6: Local streamline patterns in the neighborhood of $k$-saddles and $k / 2$ - $\partial$-saddles.

(a)

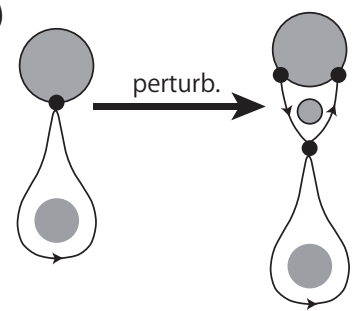

(b)

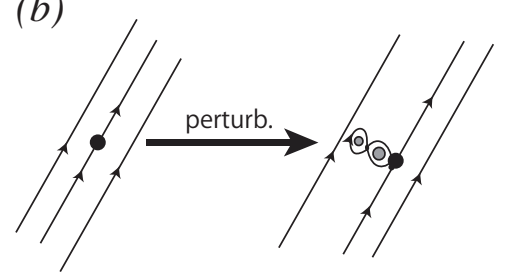

Figure 7: Unit increase in genus elements near degenerate stagnation points owing to a small perturbation: (a) 1- $\partial$-saddle and (b) 0-saddle. 


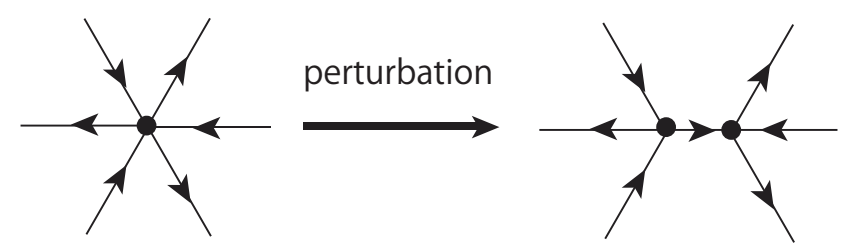

Figure 8: Perturbation of a degenerate 2-saddle yields a non-degenerate heteroclinic pair. 
(a)

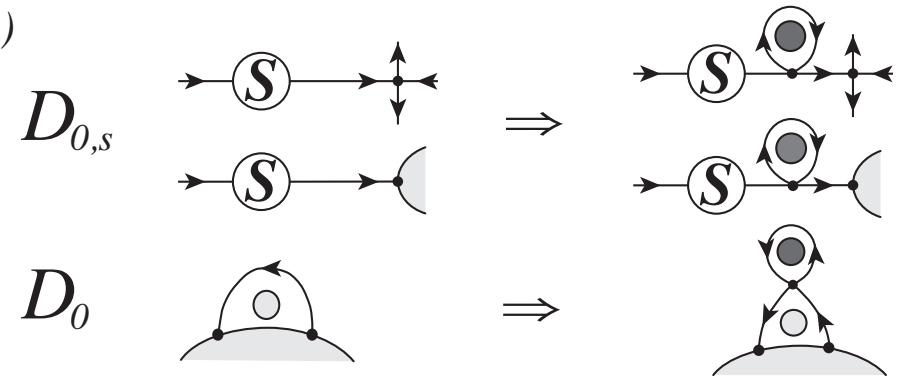

(b)

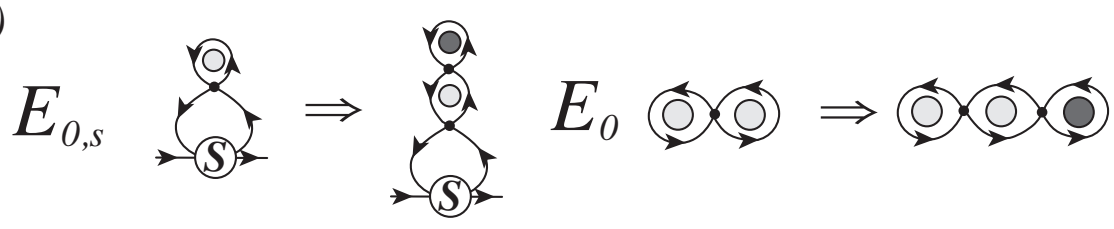

(c)

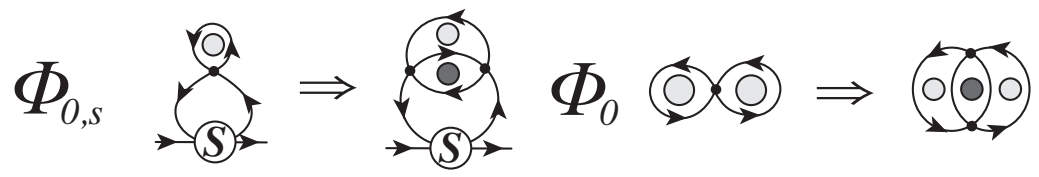

(d)

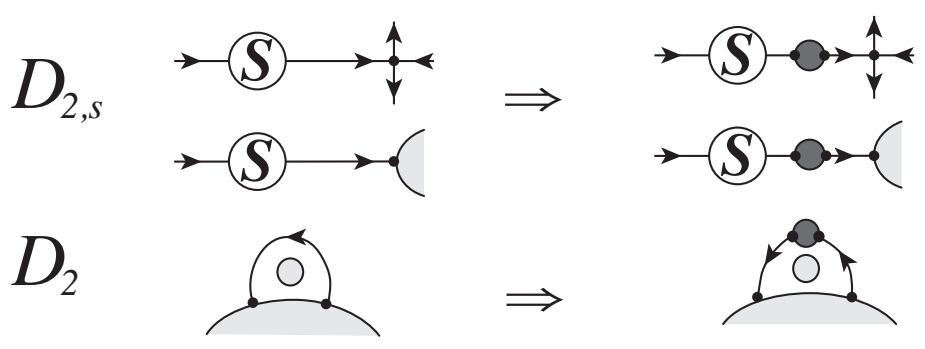

(e)

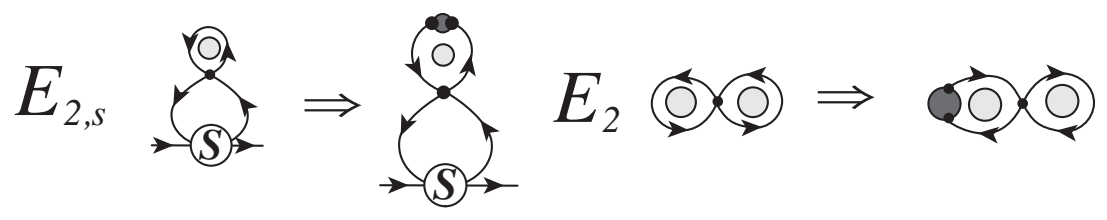

Figure 9: Ten operations creating $h$-unstable patterns with heteroclinic pairs in $\mathcal{D}_{\zeta}(M)$ by increasing the genus elements by one to the stable streamline patterns in $\mathcal{D}_{\zeta}(M-1)$. Circles filled with dark gray represent circular boundaries introduced by these operations. (a) The operations $D_{0, s}$ and $D_{0}$; (b) $E_{0, s}$ and $E_{0}$; (c) $\Phi_{0, s}$ and $\Phi_{0}$; (d) $D_{2, s}$ and $D_{2}$; (e) $E_{2, s}$ and $E_{2}$. 

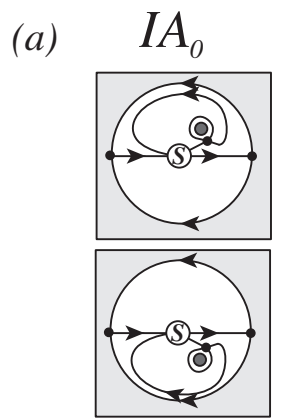

(b)
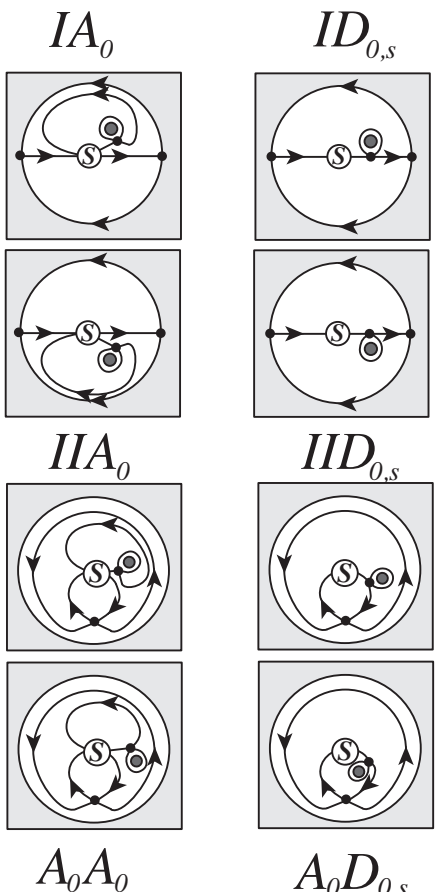

(c)
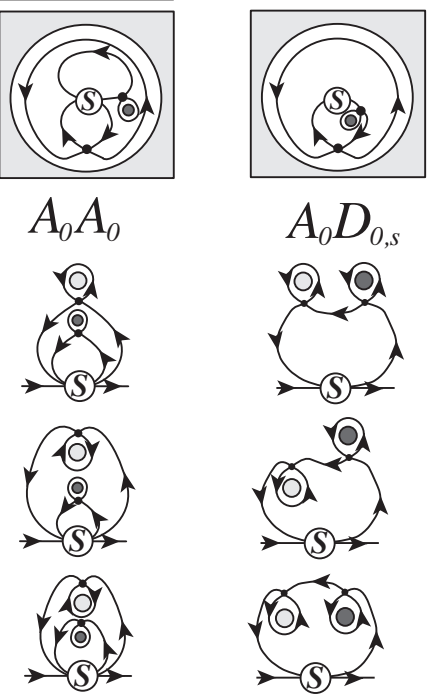

a
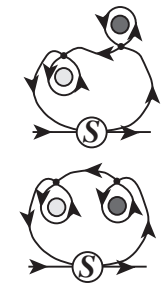

$I A$
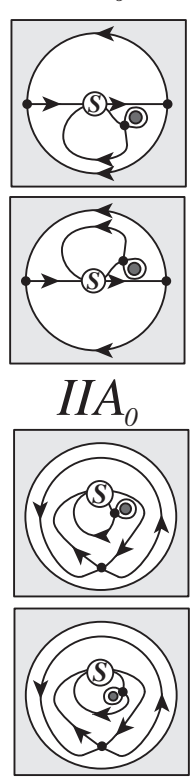

$A_{0} A_{0}$
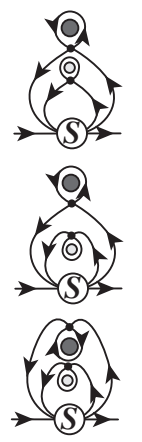

(d) $A_{2} D_{0, s}=I D_{0, s}$
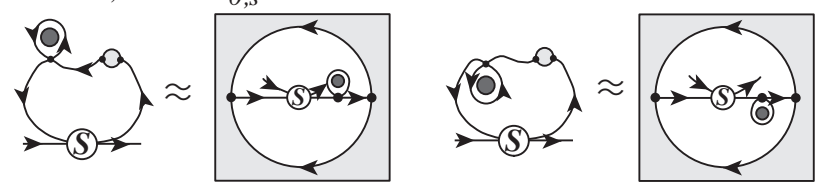

Figure 10: (a)-(c) (Center) $h$-unstable patterns generated by the operation $D_{0, s}$. (Left and right) Their perturbed structurally stable patterns with the word representations. (d) The streamline pattern $A_{0} D_{0, s}$ are topologically equivalent to $I D_{0, s}$. 
(a)
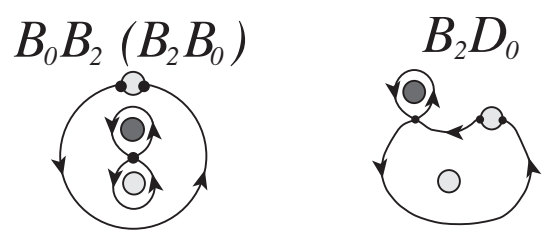

$B_{0} B_{2}\left(B_{2} B_{0}\right)$

(b)

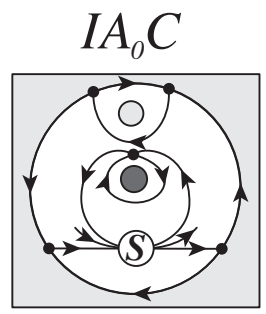

$A_{2} C D_{0} \approx I C D_{0}$
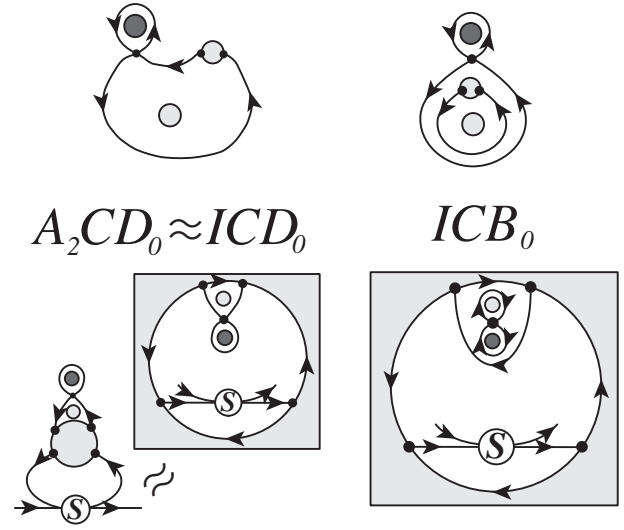

(c) $\operatorname{ICCB}_{0}\left(\mathrm{ICB}_{0} \mathrm{C}\right)$

$I C C D_{0}$
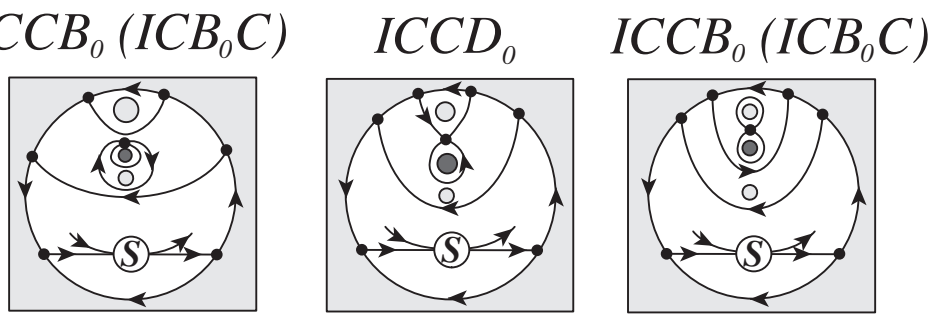

(d)
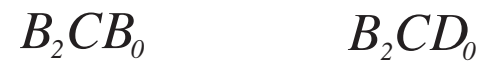

$\mathrm{B}_{2} \mathrm{CB}$

$\left(B_{0} B_{2} C, B_{2} B_{0} C\right)$

$\left(B_{0} B_{2} C, B_{2} B_{0} C\right)$
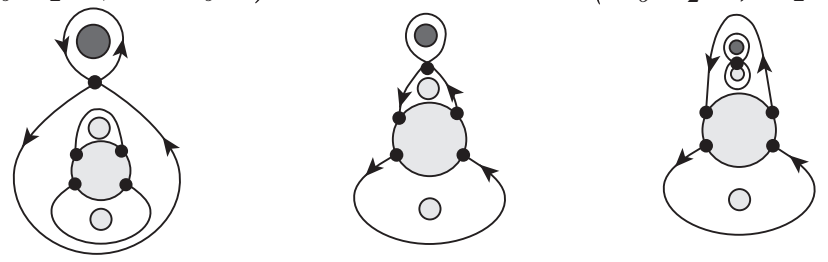

(e)
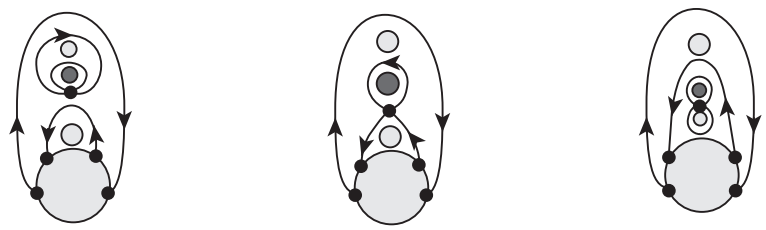

Figure 11: (Center) $h$-unstable patterns generated by the operation $D_{0}$. (Left and right) Their perturbed structurally stable patterns with the word representations. 
(a)
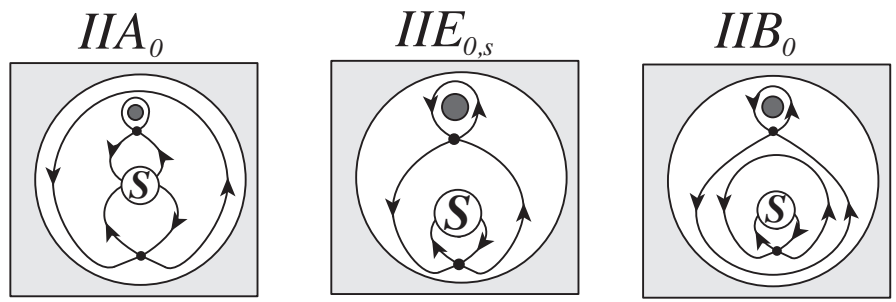

(b)

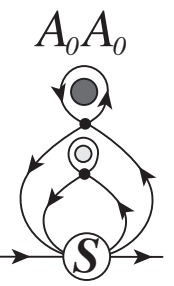

$A_{0} E_{0, s}$

$A_{0} B_{0}$

$(c)$
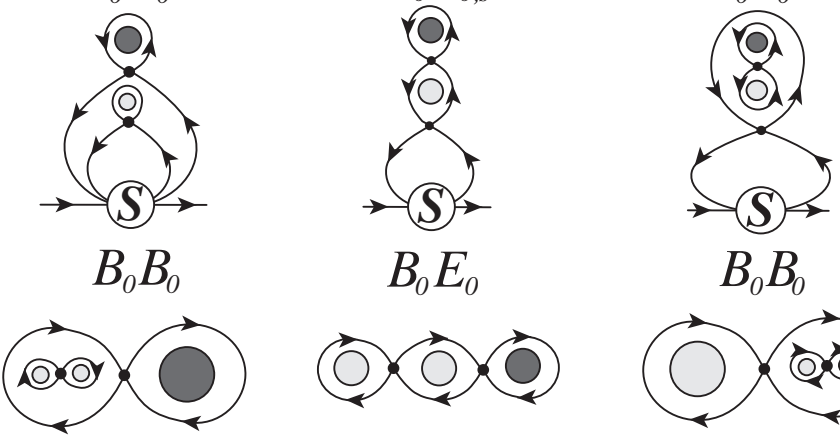

$B_{0} E_{0}$

$B_{0} B_{0}$
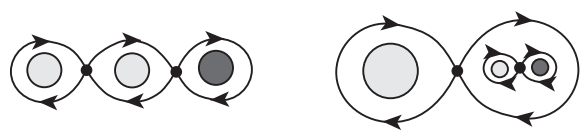

Figure 12: (Center) $h$-unstable patterns generated by the operations $E_{0, s}$ and $E_{0}$. (Left and right) Their perturbed structurally stable patterns with the word representations.

(a)
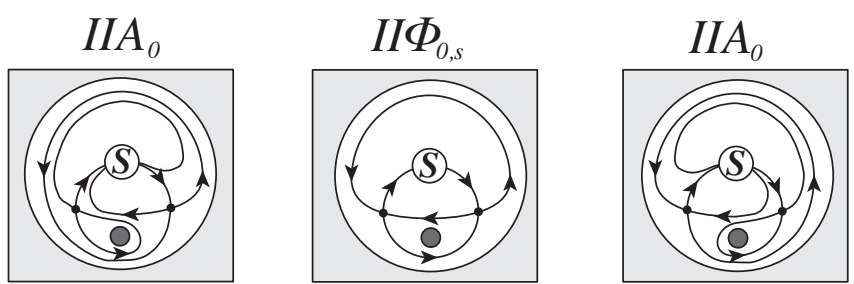

(b)
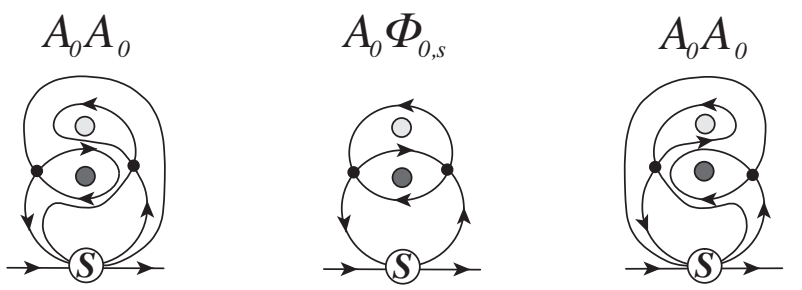

(c)
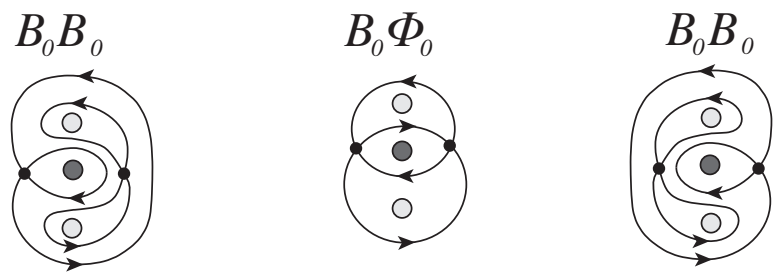

Figure 13: (Center) $h$-unstable patterns generated by the operations $\Phi_{0, s}$ and $\Phi_{0}$. (Left and right) Their perturbed structurally stable patterns with the word representations. 
(a)
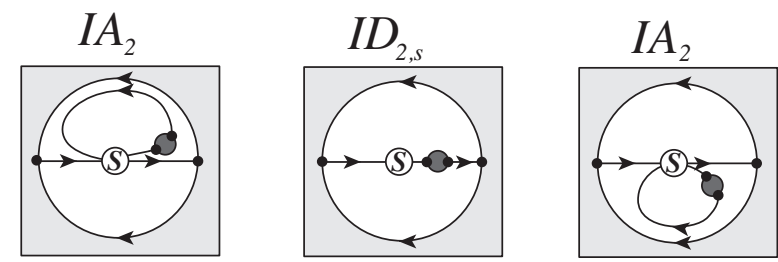

(b)
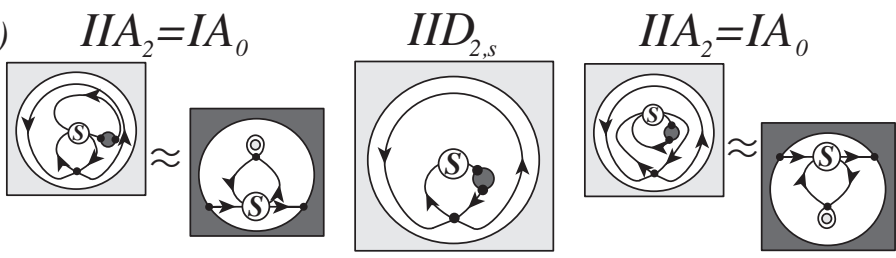

(c) $A_{0} D_{2, s}=A_{2} D_{0, s}$

(d) $A_{2} D_{2, s} \approx I D_{2, s}$
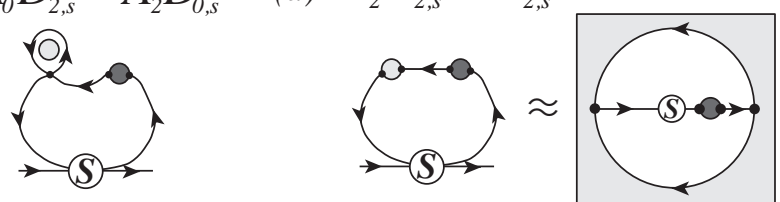

Figure 14: (a), (b) (Center) $h$-unstable patterns generated by the operation $D_{2, s}$. (Left and right) Their perturbed structurally stable patterns with the word representations. We also show topologically equivalent relations (c) $A_{0} D_{2, s}=A_{2} D_{0, s}$ and (d) $A_{2} D_{2, s} \approx I D_{2, s}$. 
(a)
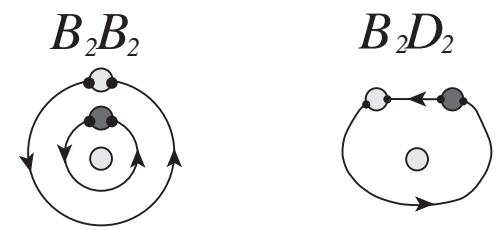

$B_{2} B_{2}$

(b)

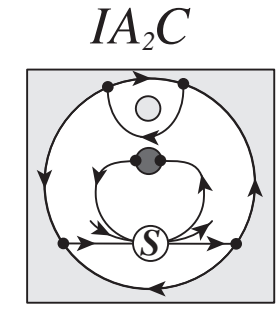

$A_{2} C D_{2} \approx I C D_{2}$

$\mathrm{ICB}_{2}$
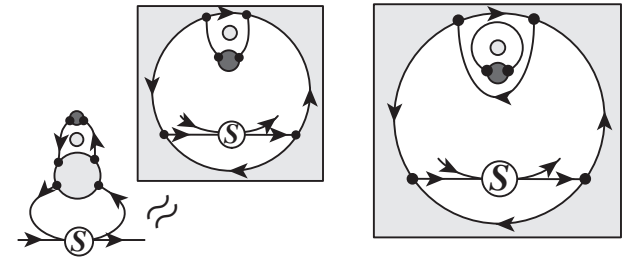

(c)
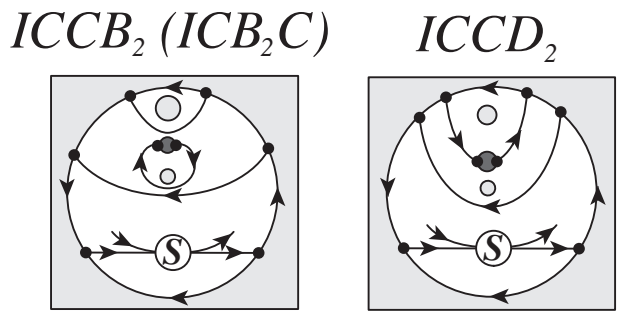

$\mathrm{ICCB}_{2}\left(\mathrm{ICB}_{2} \mathrm{C}\right)$

(d)

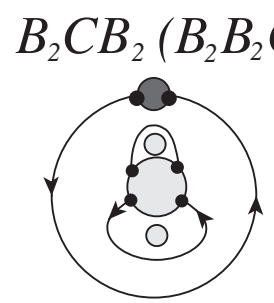

$B_{2} C D_{2}$

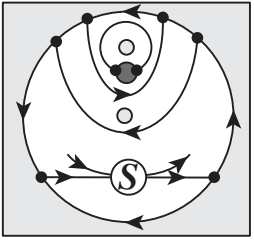

(e)

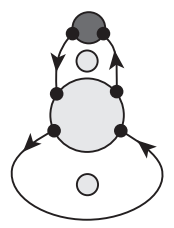

$B_{2} C B_{2}\left(B_{2} B_{2} C\right)$

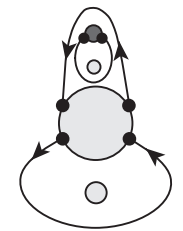

$B_{2} C C D_{2}=B_{2} C D_{2} C$
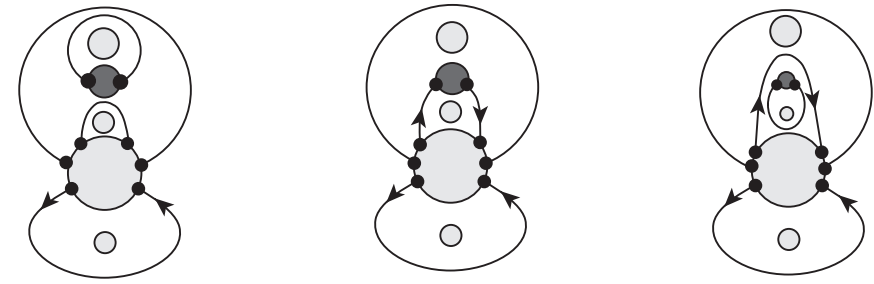

Figure 15: (Center) $h$-unstable patterns generated by the operation $D_{2}$. (Left and right) Their perturbed structurally stable patterns with the word representations. 
(a)

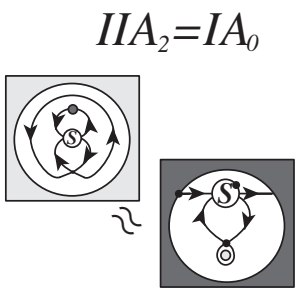

(b)

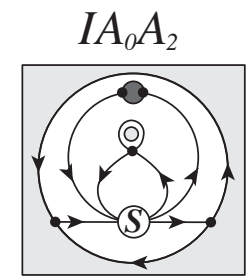

(c)

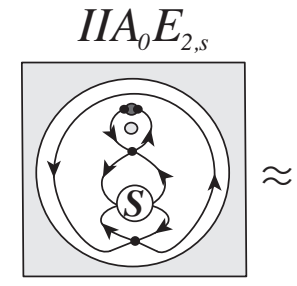

$I I E_{2, s}$

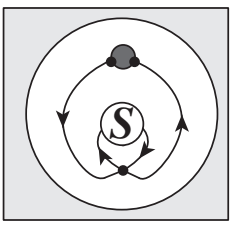

$I A_{0} E_{2, s}$

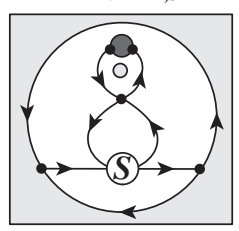

$I I E_{2, s} A_{0}$

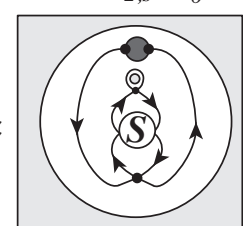

$I I B_{2}$
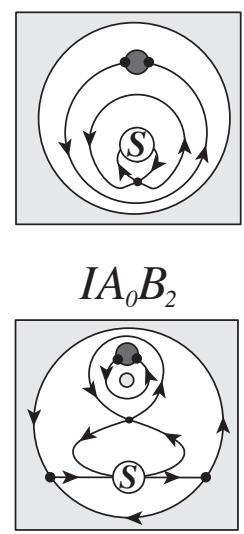

(d)

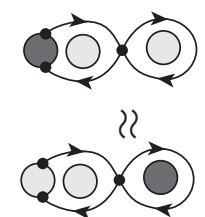

Figure 16: (a), (b) (Center) $h$-unstable patterns generated by the operations $E_{2, s}$ and $E_{2}$. (Left and right) Their perturbed structurally stable patterns with the word representations. Equivalence between the two streamline patterns: (c) $I I A_{0} E_{2, s} \approx I I E_{2, s} A_{0}$ and (d) $B_{0} E_{2}=B_{2} D_{0}$.

(a)

$\Psi_{s}(2)$<smiles>C1CCCCC1</smiles>

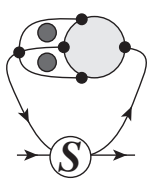

$\Psi(2)$ $(b)$<smiles>[Al]=[W]</smiles><smiles>C1CCCCC1</smiles><smiles>C1CCCCC1</smiles>

$\Xi(2 n-1)$

Figure 17: Four operations creating $h$-unstable patterns with heteroclinic connections by adding multiple genus elements to the structurally stable patterns. Circles colored with dark gray represent circular boundaries increased by the operations. (a) The operations $\Psi_{s}(2)$ and $\Psi(2)$; (b) the operations $\Xi_{s}(2 n-1)$ and $\Xi(2 n-1)$. 
(a)
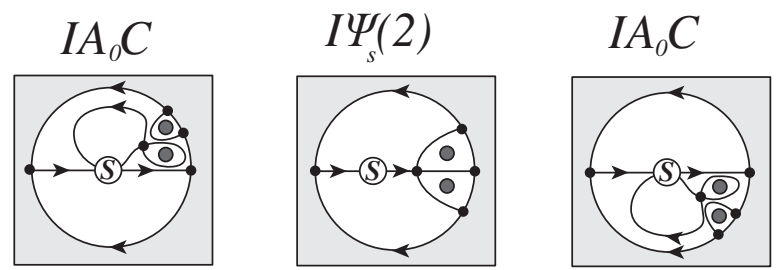

(b)

$A_{2} \Psi_{s}(2) \approx I \Psi_{s}(2)$
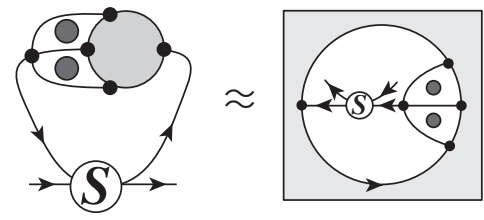

(c)

$B_{0} B_{2} C$

$B_{2} \Psi(2)$

$\left(B_{2} C B_{0}, B_{2} B_{0} C\right)$

$B_{0} B_{2} C$
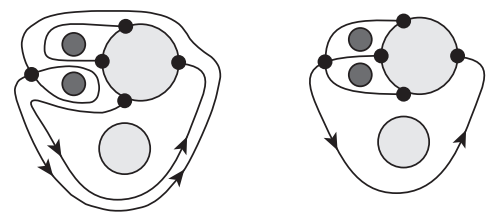

$\left(B_{2} C B_{0}, B_{2} B_{0} C\right)$

Figure 18: (a), (c) (Center) $h$-unstable patterns generated by the operations $\Psi_{s}(2)$ and $\Psi(2)$. (Left and right) Their perturbed structurally stable patterns with the word representations. (b) The streamline pattern $A_{2} \Psi_{s}(2)$ is equivalent to $I \Psi_{s}(2)$. 
(a)
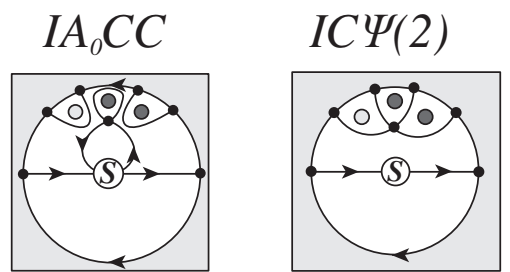

$I C C B_{0}\left(\mathrm{ICB}_{0} \mathrm{C}\right)$

(b)

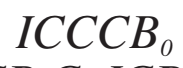

$\operatorname{ICC} \Psi(2)$

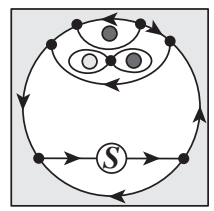

$\left(I C C B_{0} C, I C B_{0} C C\right)$
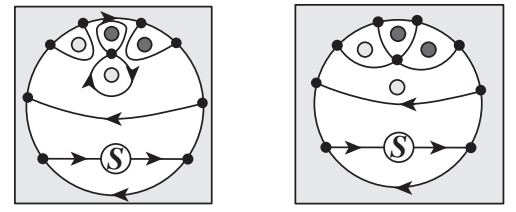

$I C C C B_{0}$
$\left(I C C B_{0} C, I C B_{0} C C\right)$

(c) $B_{2} C C B_{0}\left(B_{0} B_{2} C C\right) \quad B_{2} C \Psi(2)$

$\left(B_{2} B_{0} C C, B_{2} C B_{0} C\right)$
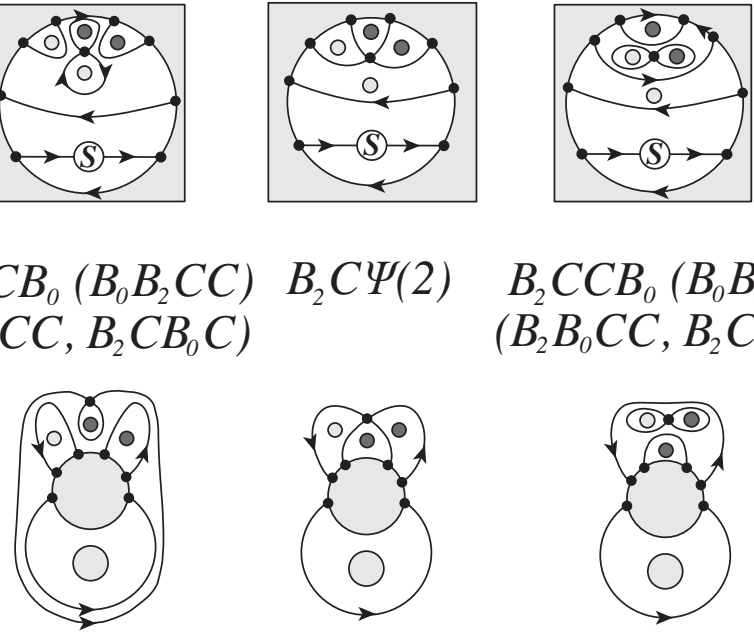

$B_{2} C C B_{0}\left(B_{0} B_{2} C C\right)$

$\left(B_{2} B_{0} C C, B_{2} C B_{0} C\right)$

(d)

$B_{2} C C \Psi(2)=B_{2} C \Psi(2) C$
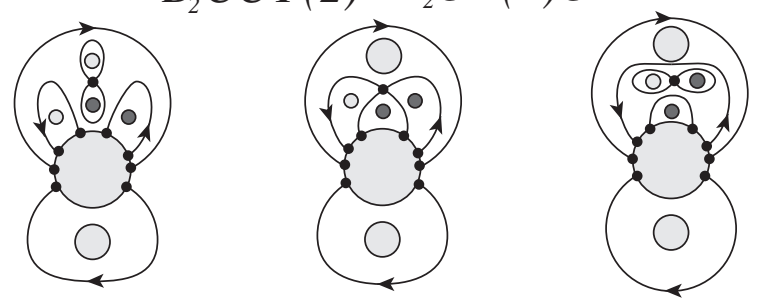

Figure 19: (a)-(c) (Center) $h$-unstable patterns generated by the operations $\Psi(2)$. (Left and right) Their perturbed structurally stable patterns with the word representations. (d) The streamline pattern $B_{2} C C \Psi(2)$ is equivalent to $B_{2} C \Psi(2) C$. 
(a)
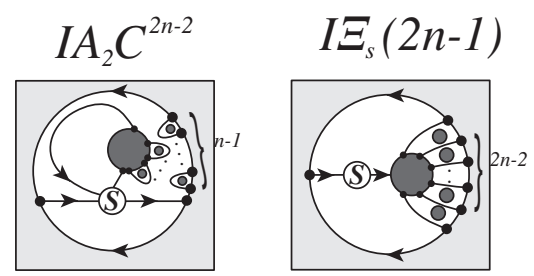

$I A_{2} C^{2 n-2}$

(b)
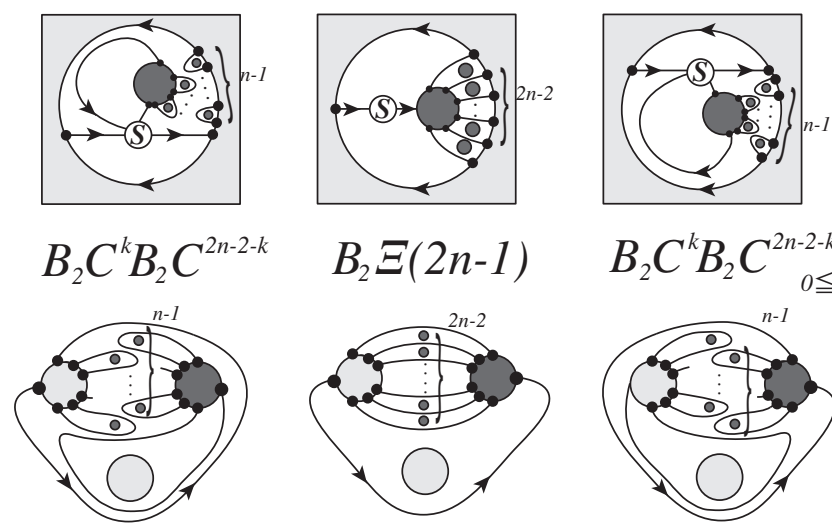

$B_{2} C^{k} B_{2} C_{0 \leqq k \leqq n-1}^{2 n-2-k}$

(c)
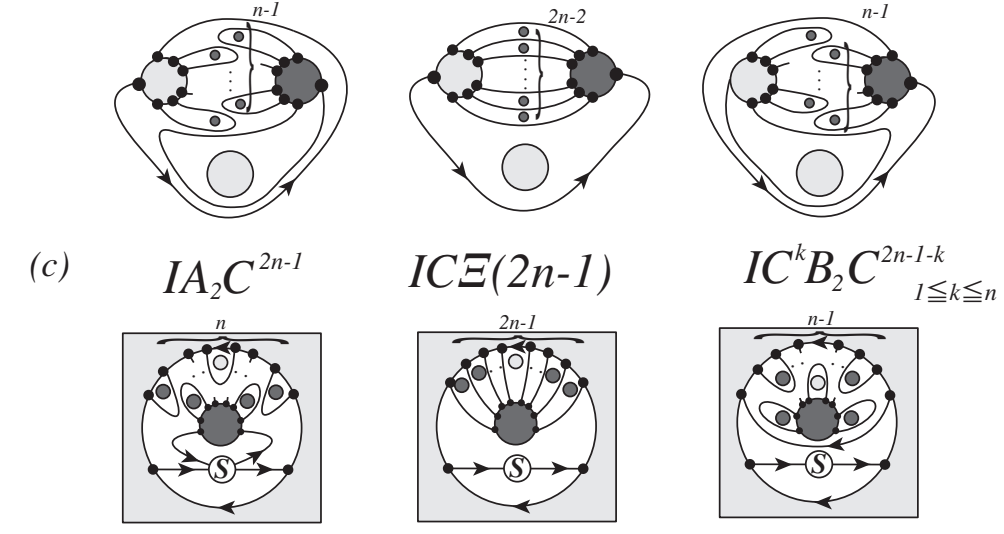

(d)
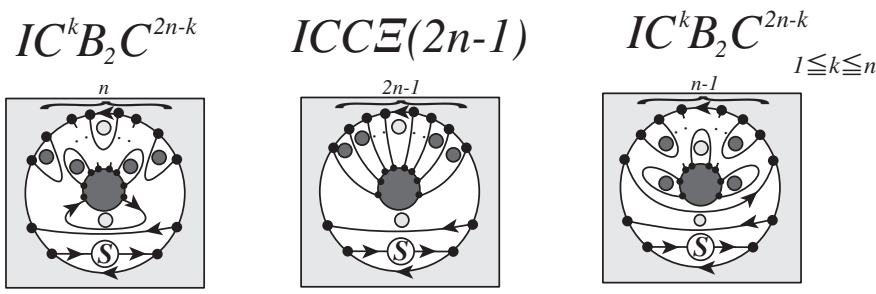

Figure 20: (Center) $h$-unstable patterns generated by the operations $\Xi_{s}(2)$ and $\Xi(2)$. (Left and right) Their perturbed structurally stable patterns with the word representations.

(a)

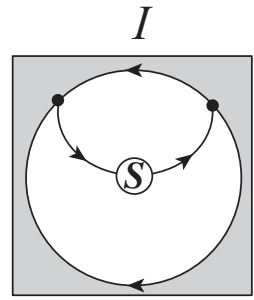

(b)

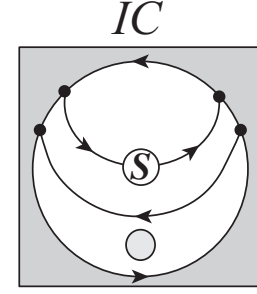

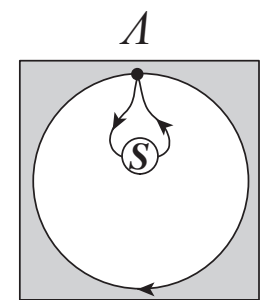

$\Lambda C$

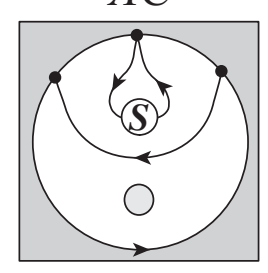

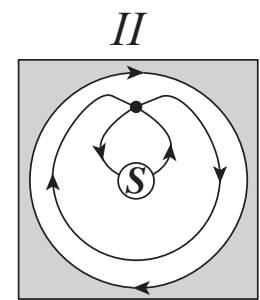

$I I B_{2}$

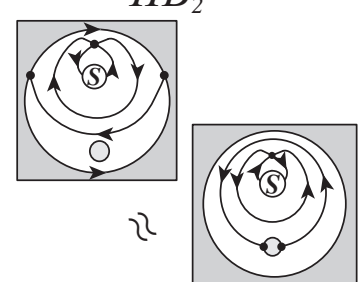

Figure 21: (Center) $p$-unstable patterns $\Lambda$ and $\Lambda C$. (Left and right) Their perturbed structurally stable patterns in $\chi_{1}^{r}$. 


\begin{tabular}{|c|c|c|c|c|c|}
\hline operation & $X$ & $S_{1}$ & $S_{2}$ & $d$ & Figure \\
\hline \multirow[t]{3}{*}{ 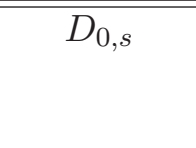 } & $\overline{I I D_{0, s}\left(\approx A_{2} D_{0, s}\right)}$ & $\overline{I I A_{0}\left(\approx A_{0} A_{2}\right)}$ & $\overline{I A_{0}\left(\approx A_{0} A_{2}\right)}$ & $\overline{0}$ & $\overline{10(\mathrm{a})}$ \\
\hline & $I I D_{0, s}$ & $I I A_{0}$ & $I I A_{0}$ & $\mathbf{0}$ & $10(b)$ \\
\hline & $A_{0} D_{0, s}$ & $A_{0} A_{0}$ & $A_{0} A_{0}$ & $\mathbf{0}$ & $10(c)$ \\
\hline \multirow[t]{5}{*}{$D_{0}$} & $B_{2} D_{0}$ & $B_{0} B_{2}, B_{2} B_{0}$ & $B_{0} B_{2}, B_{2} B_{0}$ & 0 & $11(\mathrm{a})$ \\
\hline & $I C D_{0}\left(\approx A_{2} C D_{0}\right)$ & $I A_{0} C\left(\approx A_{0} A_{2} C\right)$ & $I C B_{0}\left(\approx A_{2} C B_{0}\right)$ & $(1,0,-1,0,0)$ & $11(b)$ \\
\hline & $I C C D_{0}$ & $I C C B_{0}, I C B_{0} C$ & $I C C B_{0}, I C B_{0} C$ & 0 & $11(\mathrm{c})$ \\
\hline & $B_{2} C D_{0}$ & $B_{2} C B_{0}$ & $B_{2} C B_{0}$ & 0 & $11(\mathrm{~d})(\mathrm{e})$ \\
\hline & & $B_{0} B_{2} C, B_{2} B_{0} C$ & $B_{0} B_{2} C, B_{2} B_{0} C$ & & \\
\hline \multirow[t]{2}{*}{$E_{0, s}$} & $I I E_{0, s}$ & $I I A_{0}$ & $I I B_{0}$ & $(1,0,-1,0,0)$ & $12(\mathrm{a})$ \\
\hline & $A_{0} E_{0, s}$ & $A_{0} A_{0}$ & $A_{0} B_{0}$ & $(1,0,-1,0,0)$ & $12(\mathrm{~b})$ \\
\hline$E_{0}$ & $B_{0} E_{0}$ & $B_{0} B_{0}$ & $B_{0} B_{0}$ & 0 & $12(\mathrm{c})$ \\
\hline \multirow[t]{2}{*}{$\Phi_{0, s}$} & $I I \Phi_{0, s}$ & $I I A_{0}$ & $I I A_{0}$ & 0 & $13(\mathrm{a})$ \\
\hline & $A_{0} \Phi_{0, s}$ & $A_{0} A_{0}$ & $A_{0} A_{0}$ & 0 & $13(\mathrm{~b})$ \\
\hline$\Phi_{0}$ & $B_{0} \Phi_{0}$ & $B_{0} B_{0}$ & $B_{0} B_{0}$ & 0 & $13(\mathrm{c})$ \\
\hline \multirow[t]{3}{*}{$D_{2, s}$} & $I D_{2, s}\left(\approx A_{2} D_{2, s}\right)$ & $I A_{2}\left(\approx A_{2} A_{2}\right)$ & $I A_{2}\left(\approx A_{2} A_{2}\right)$ & 0 & $14(\mathrm{a})$ \\
\hline & $I I D_{2, s}$ & $I A_{0}$ & $I A_{0}$ & 0 & $14(b)$ \\
\hline & $A_{0} D_{2, s}\left(=A_{2} D_{0, s}\right)$ & $A_{0} A_{2}$ & $A_{0} A_{2}$ & $\mathbf{0}$ & $14(\mathrm{c})$ \\
\hline \multirow[t]{4}{*}{$D_{2}$} & $B_{2} D_{2}$ & $B_{2} B_{2}$ & $B_{2} B_{2}$ & 0 & $15(\mathrm{a})$ \\
\hline & $I C D_{2}\left(\approx A_{2} C D_{2}\right)$ & $I A_{2} C\left(\approx A_{2} A_{2} C\right)$ & $I C B_{2}\left(\approx A_{2} C B_{2}\right)$ & $(0,1,0,-1,0)$ & $15(b)$ \\
\hline & $I C C D_{2}$ & $I C C B_{2}, I C B_{2} C$ & $I C C B_{2}, I C B_{2} C$ & 0 & $15(\mathrm{c})$ \\
\hline & $B_{2} C D_{2}$ & $B_{2} C B_{2}, B_{2} B_{2} C$ & $B_{2} C B_{2}, B_{2} B_{2} C$ & 0 & $15(\mathrm{~d})(\mathrm{e})$ \\
\hline \multirow[t]{2}{*}{$E_{2, s}$} & $I I E_{2, s}$ & $I A_{0}$ & $I I B_{2}$ & $(0,1,0,-1,0)$ & $16(\mathrm{a})$ \\
\hline & $I A_{0} E_{2, s}$ & $I A_{0} A_{2}$ & $I A_{0} B_{2}$ & $(0,1,0,-1,0)$ & $16(\mathrm{~b})$ \\
\hline$E_{2}$ & $B_{0} E_{2}\left(=B_{2} D_{0}\right)$ & $B_{0} B_{2}, B_{2} B_{0}$ & $B_{0} B_{2}, B_{2} B_{0}$ & 0 & $16(d)$ \\
\hline$\Psi_{s}(2)$ & $I \Psi_{s}(2)\left(\approx A_{2} \Psi_{s}(2)\right)$ & $I A_{0} C\left(\approx A_{0} A_{2} C\right)$ & $I A_{0} C\left(\approx A_{0} A_{2} C\right)$ & 0 & $18(\mathrm{a})$ \\
\hline \multirow[t]{6}{*}{$\Psi(2)$} & $B_{2} \Psi(2)$ & $\begin{array}{c}B_{0} B_{2} C \\
B_{2} C B_{0}, B_{2} B_{0} C\end{array}$ & $\begin{array}{c}B_{0} B_{2} C \\
B_{2} C B_{0}, B_{2} B_{0} C\end{array}$ & 0 & $18(\mathrm{c})$ \\
\hline & $I C \Psi(2)$ & $I A_{0} C C$ & $I C C B_{0}, I C B_{0} C$ & $(1,0,-1,0,0)$ & $19(\mathrm{a})$ \\
\hline & $I C C \Psi(2)$ & $I C C C B_{0}$ & $I C C C B_{0}$ & 0 & $19(b)$ \\
\hline & & $I C C B_{0} C, I C B_{0} C C$ & $I C C B_{0} C, I C B_{0} C C$ & & \\
\hline & $B_{2} C \Psi(2)$ & $B_{2} C C B_{0}, B_{0} B_{2} C C$ & $B_{2} C C B_{0}, B_{0} B_{2} C C$ & 0 & $19(\mathrm{c})(\mathrm{d})$ \\
\hline & & $B_{2} B_{0} C C, B_{2} C B_{0} C$ & $B_{2} B_{0} C C, B_{2} C B_{0} C$ & & \\
\hline$\Xi_{s}(2 n-1)$ & $\begin{array}{c}I \Xi_{s}(2 n-1) \\
\left(\approx A_{2} \Xi_{s}(2 n-1)\right)\end{array}$ & $\begin{aligned} & I A_{2} C^{2 n-2} \\
(\approx & \left.A_{2} A_{2} C^{2 n-2}\right)\end{aligned}$ & $\begin{array}{c}I A_{2} C^{2 n-2} \\
\left(\approx A_{2} A_{2} C^{2 n-2}\right)\end{array}$ & 0 & $20(\mathrm{a})$ \\
\hline \multirow[t]{3}{*}{$\Xi(2 n-1)$} & $B_{2} \Xi(2 n-1)$ & $B_{2} C^{k} B_{2} C^{2 n-2-k}$ & $B_{2} C^{k} B_{2} C^{2 n-2-k}$ & 0 & $20(b)$ \\
\hline & $I C \Xi(2 n-1)$ & $I A_{2} C^{2 n-1}$ & $I C^{k} B_{2} C^{2 n-1-k}$ & $(0,1,0,-1,0)$ & $20(\mathrm{c})$ \\
\hline & $I C C \Xi(2 n-1)$ & $I C^{k} B_{2} C^{2 n-k}$ & $I C^{k} B_{2} C^{2 n-k}$ & 0 & $20(d)$ \\
\hline
\end{tabular}

Table 1: List of possible transitions between structurally stable Hamiltonian vector fields through a marginal $h$-unstable vector field. The $h$-unstable streamline pattern $X$ with heteroclinic connections is a transient state between the two structurally stable vector fields represented by $W_{1}$ and $W_{2}$, if $S_{1} \subseteq W_{1}, S_{2} \subseteq W_{2}$ and $\operatorname{ind}_{*}\left(W_{1}\right)-\operatorname{ind}_{*}\left(W_{2}\right)=\boldsymbol{d}$ for $* \in\{O, I, I I\}$ are satisfied. 
(a)

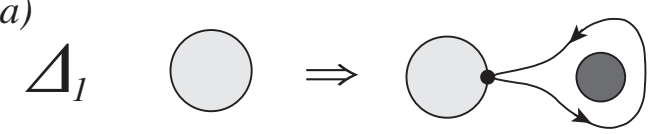

(c)

$M_{1, o}$

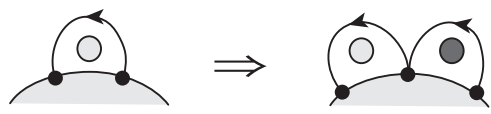

(b)

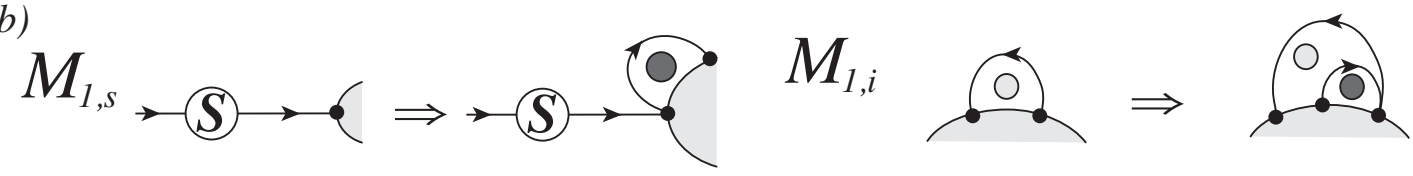

Figure 22: Four operations creating $p$-unstable orbits with a pinching point. (a) The operations $\Delta_{1}$; (b) $M_{1, s} ;$ (c) $M_{1, o}$ and $M_{1, i}$.

(a)

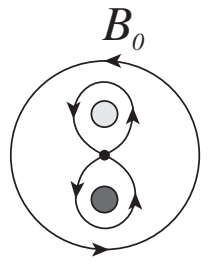

(b)

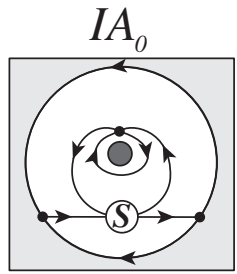

(c)

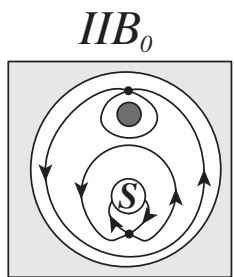

(d)

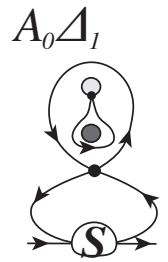

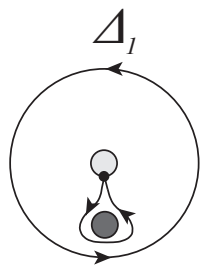
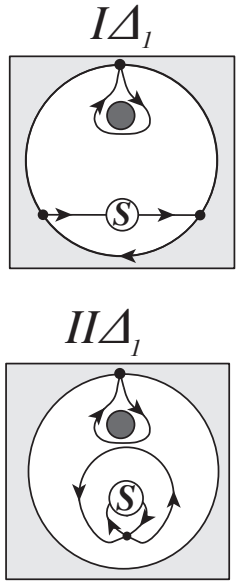

(e) $\quad B_{0} \Delta_{l}$

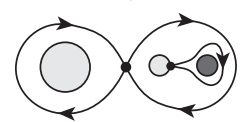

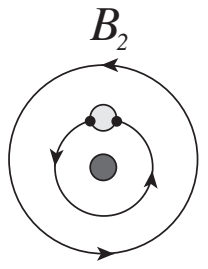

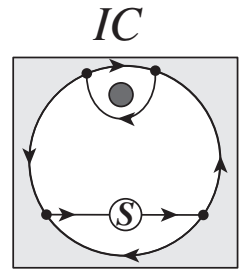

$I I B_{2}$
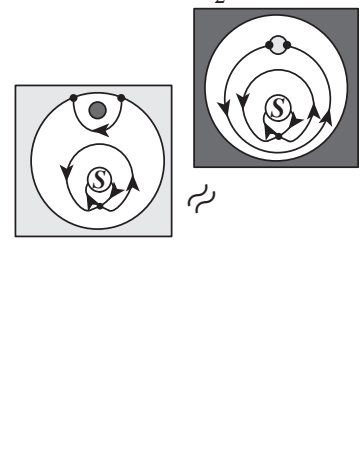

Figure 23: (a)-(c) (Center) $p$-unstable patterns generated by the operation $\Delta_{1}$. (Left and right) Their perturbed structurally stable patterns with the word representations. (d)-(e) The streamline patterns $A_{0} \Delta_{1}$ and $B_{0} \Delta_{1}$. 
(a)

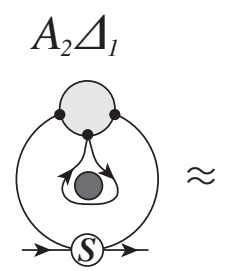

(c)

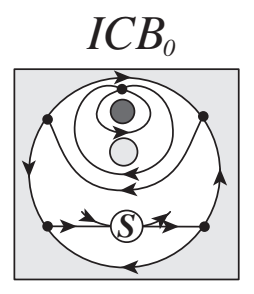

$I \Delta_{1}$

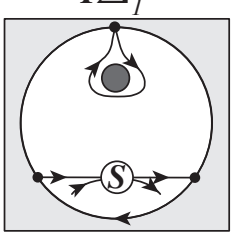

$A_{2} C \Delta_{1} \approx I C \Delta_{1}$

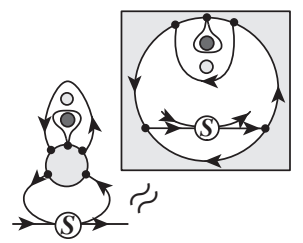

(b)
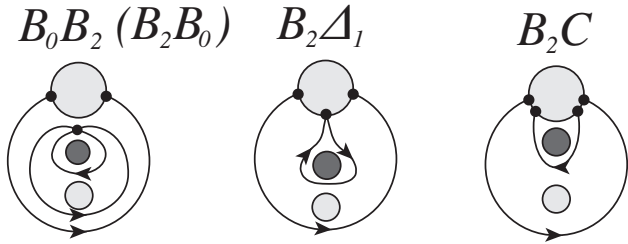

(d)

$B_{2} C \Delta_{l}=B_{2} \Delta_{l} C$
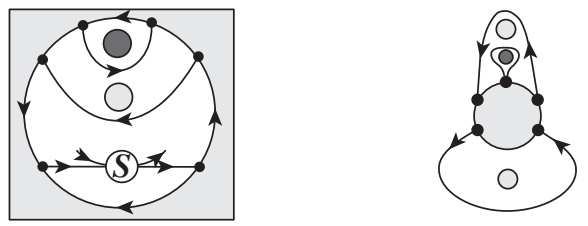

Figure 24: (a) Topological equivalence $A_{2} \Delta_{1} \approx I \Delta_{1}$. (b)-(c) (Center) p-unstable patterns generated by the operation $\Delta_{1}$. (Left and right) Their perturbed structurally stable patterns with the word representations. (d) $B_{2} C \Delta_{1}$ is equivalent to $B_{2} \Delta_{1} C$.

(a)

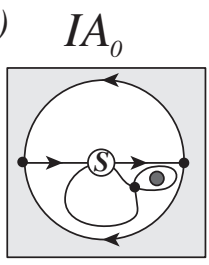

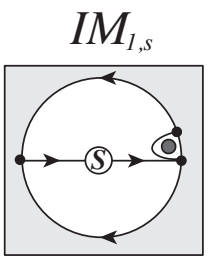

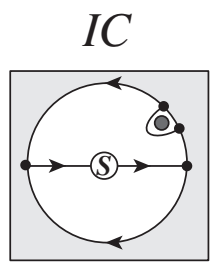

(b) $A_{2} M_{1, s}$
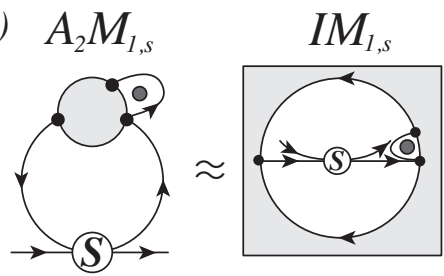

Figure 25: (a) (Center) $p$-unstable unstable patterns generated by the operation $M_{1, s}$. (Left and right) Their perturbed structurally stable patterns with the word representations. (b) The streamline pattern $A_{2} M_{1, s}$ is topologically equivalent to $I M_{1, s}$. 
(a)

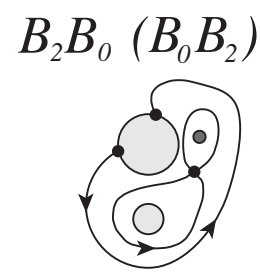

$B_{2} M_{1, o}$

$B_{2} C$
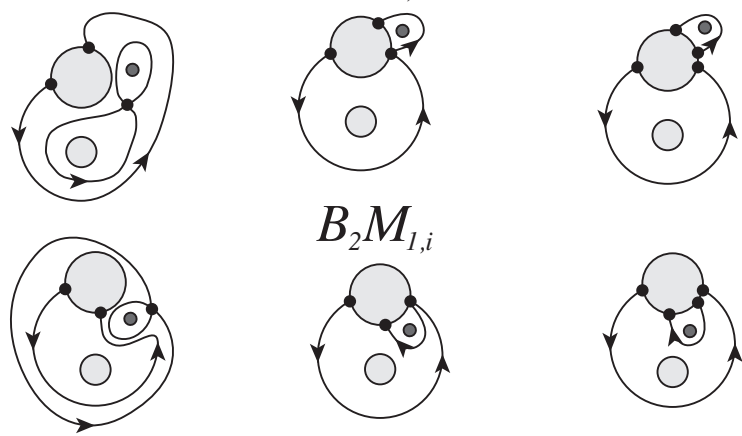

(b)
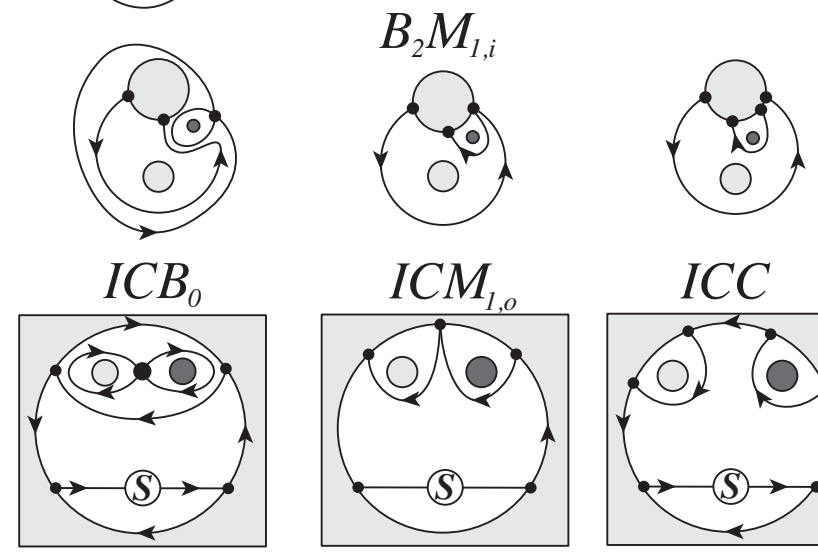

$I A_{0} C$
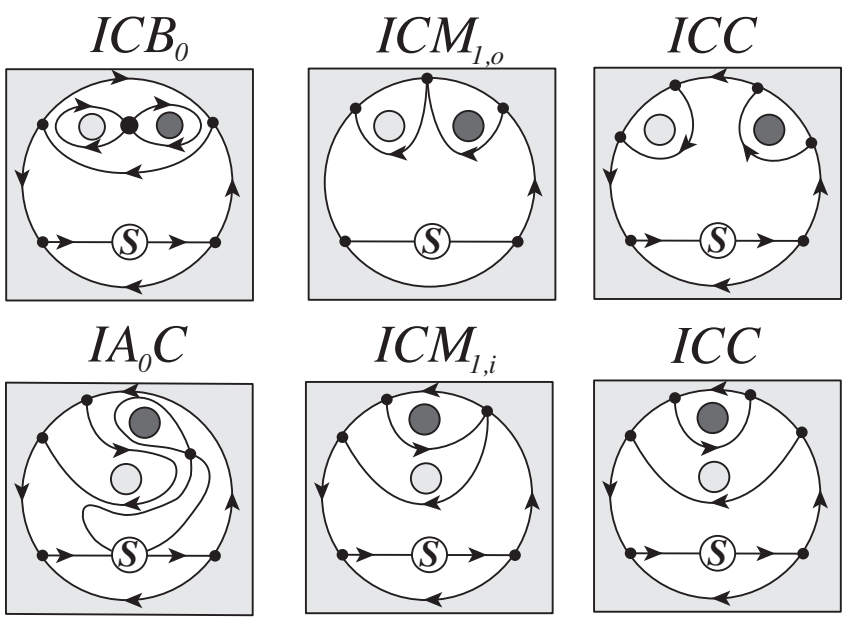

ICC

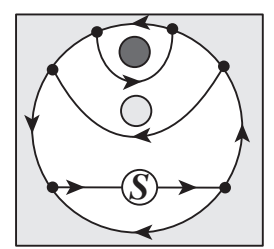

(c)
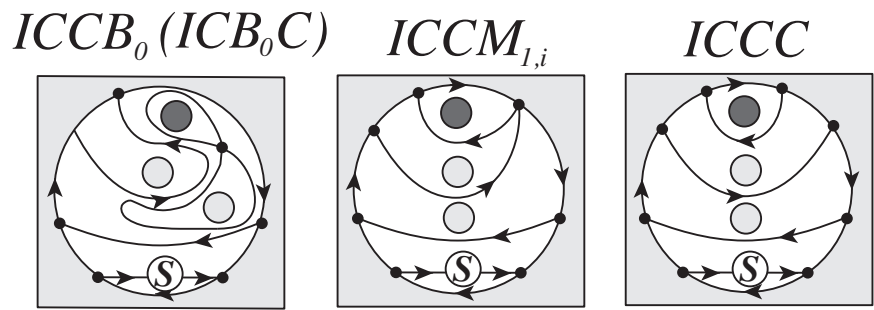

Figure 26: (Center) $p$-unstable patterns generated by the operations $M_{1, i}$ and $M_{1, o}$. (Left and right) Their perturbed structurally stable patterns with the word representations. 


\begin{tabular}{cccccc} 
operation & $X$ & $S_{1}$ & $S_{2}$ & $\boldsymbol{d}$ & Figure \\
\hline \hline$\Lambda$ & $\Lambda$ & $I$ & $I I$ & $(-1,1,0,0,0)$ & $21(\mathrm{a})$ \\
& $\Lambda C$ & $I C$ & $I I B_{2}$ & $(-1,1,0,-1,1)$ & $21(\mathrm{~b})$ \\
\hline$\Delta_{1}$ & $\Delta_{1}$ & $B_{0}$ & $B_{2}$ & $(0,0,1,-1,0)$ & $23(\mathrm{a})$ \\
& $I \Delta_{1}\left(\approx A_{2} \Delta_{1}\right)$ & $I A_{0}\left(\approx A_{0} A_{2}\right)$ & $I C\left(\approx A_{2} C\right)$ & $(1,0,0,0,-1)$ & $23(\mathrm{~b})$ \\
& $I I \Delta_{1}$ & $I I B_{0}$ & $I I B_{2}$ & $(0,0,1,-1,0)$ & $23(\mathrm{c})$ \\
& $B_{2} \Delta_{1}$ & $B_{0} B_{2}, B_{2} B_{0}$ & $B_{2} C$ & $(0,0,1,0,-1)$ & $24(\mathrm{~b})$ \\
& $I C \Delta_{1}\left(\approx A_{2} C \Delta_{1}\right)$ & $I C B_{0}\left(\approx A_{2} C B_{0}\right)$ & $I C C\left(\approx A_{2} C C\right)$ & $(0,0,1,0,-1)$ & $24(\mathrm{c})$ \\
\hline$M_{1, s}$ & $I M_{1, s}\left(\approx A_{2} M_{1, s}\right)$ & $I A_{0}\left(\approx A_{0} A_{2}\right)$ & $I C\left(\approx A_{2} C\right)$ & $(1,0,0,0,-1)$ & $25(\mathrm{a})$ \\
\hline$M_{1, o}$ & $B_{2} M_{1, o}$ & $B_{2} B_{0}, B_{0} B_{2}$ & $B_{2} C$ & $(0,0,1,0,-1)$ & $26(\mathrm{a})$ \\
& $I C M_{1, o}$ & $I C B_{0}$ & $I C C$ & $(0,0,1,0,-1)$ & $26(\mathrm{~b})$ \\
\hline$M_{1, i}$ & $B_{2} M_{1, i}$ & $B_{2} B_{0}$ & $B_{2} C$ & $(0,0,1,0,-1)$ & $26(\mathrm{a})$ \\
& $I C M_{1, i}$ & $I A_{0} C$ & $I C C$ & $(1,0,0,0,-1)$ & $26(\mathrm{~b})$ \\
& $I C C M_{1, i}$ & $I C C B_{0}, I C B_{0} C$ & $I C C C$ & $(0,0,1,0,-1)$ & $26(\mathrm{c})$ \\
\hline
\end{tabular}

Table 2: List of possible transitions between structurally stable Hamiltonian vector fields through a transient $p$-unstable vector field. The $p$-unstable streamline pattern $X$ is a transient state between the two structurally stable vector fields represented by $W_{1}$ and $W_{2}$, if $S_{1} \subseteq W_{1}, S_{2} \subseteq W_{2}$ and $\operatorname{ind}_{*}\left(W_{1}\right)-\operatorname{ind}_{*}\left(W_{2}\right)=\boldsymbol{d}$ for $* \in\{O, I, I I\}$.

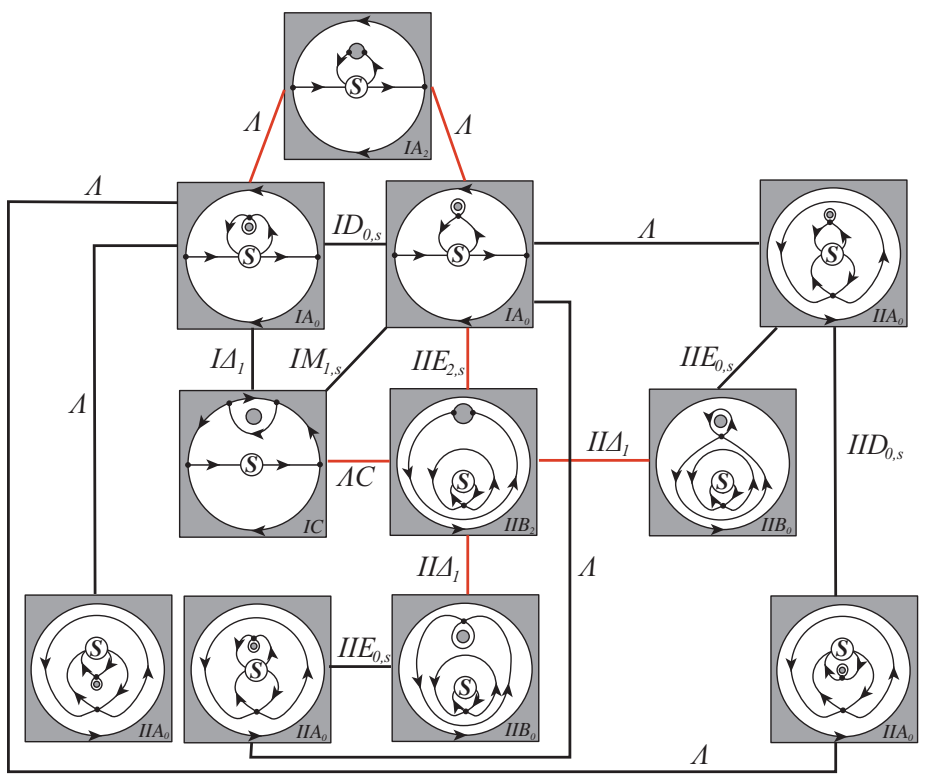

Figure 27: Transition graph between structurally stable Hamiltonian vector fields with the 1source-sink point in the domain $\mathcal{D}_{\zeta}(1)$. 


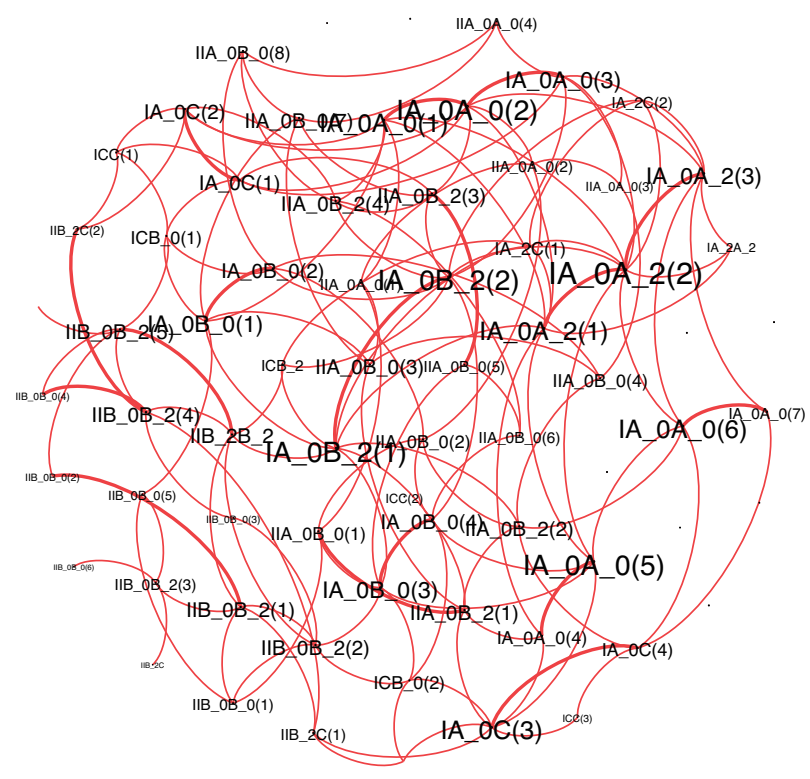

Figure 28: Transition graph between structurally stable Hamiltonian vector fields with the 1source-sink point in the domain $\mathcal{D}_{\zeta}(2)$. The labels of the nodes represent the maximal words of the structurally stable Hamiltonian vector fields. The labels of the edges are not shown.

\section{(a) $I$}

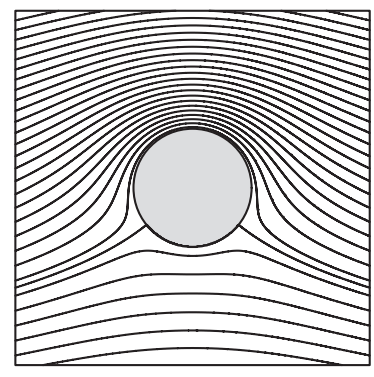

(b) $I I$

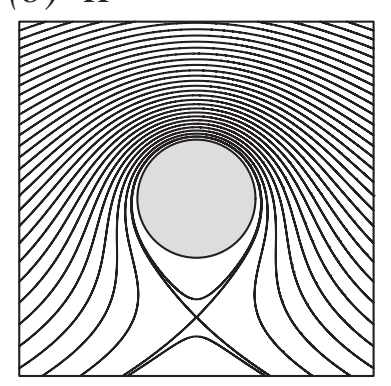

(c) $\Lambda$

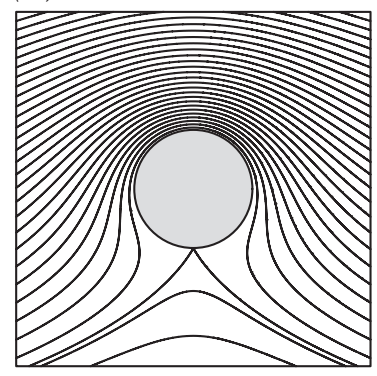

Figure 29: Streamline topologies for the uniform flow around a circle represented by (4) with (a) $U=1$ and $\Gamma=-2.5 \pi$, (b) $U=1$ and $\Gamma=-5.5 \pi$, (c) $U=1$ and $\Gamma=-4 \pi$. 

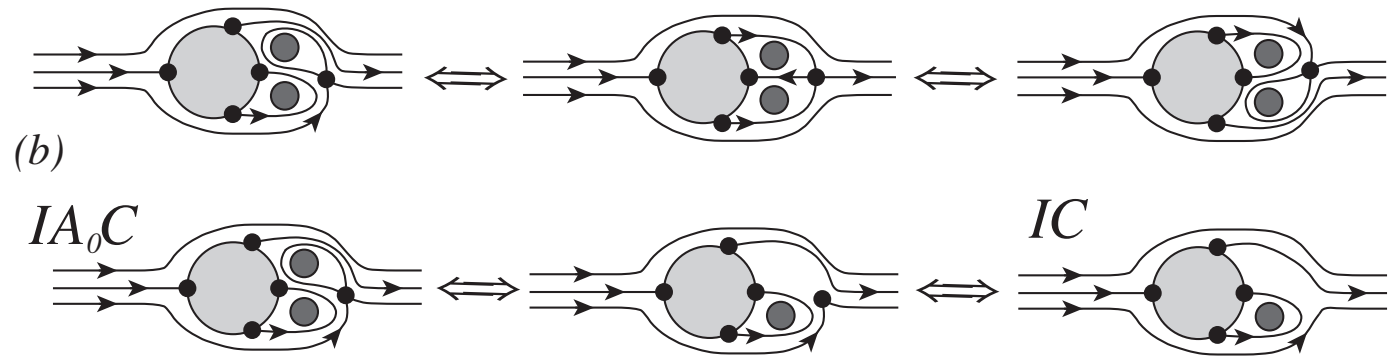

Figure 30: Schematic scenarios of topological streamline transitions behind a circular boundary in the uniform flow obtained by the bifurcation analysis[7]. (a) Periodic change between the mirror symmetric streamline patterns for the Reynolds number around 45; (b) Transition between streamline patterns owing to the saddle-center bifurcation for the Reynolds number around 100. 
(a) $t=5.5$
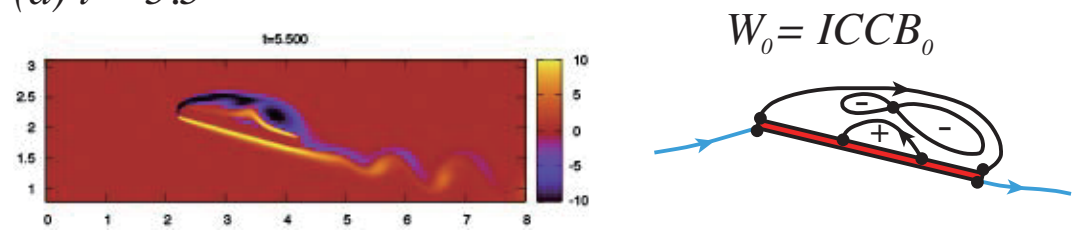

(b) $t=6.6$
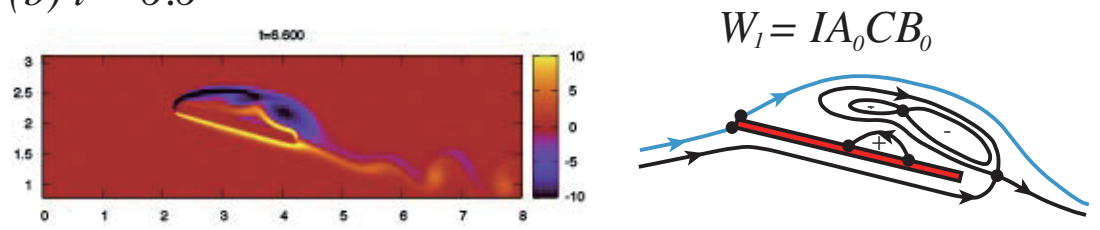

(c) $t=7.7$
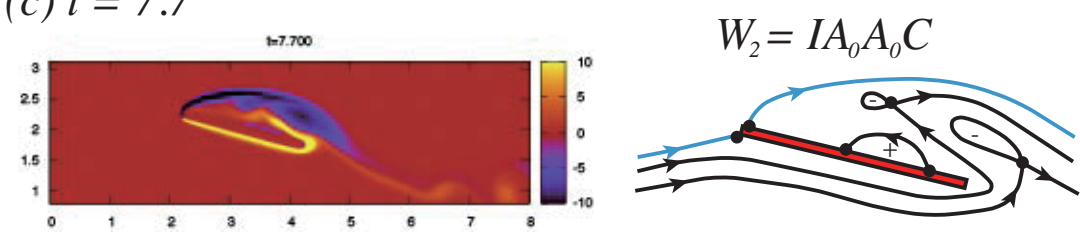

(d) $t=8.8$
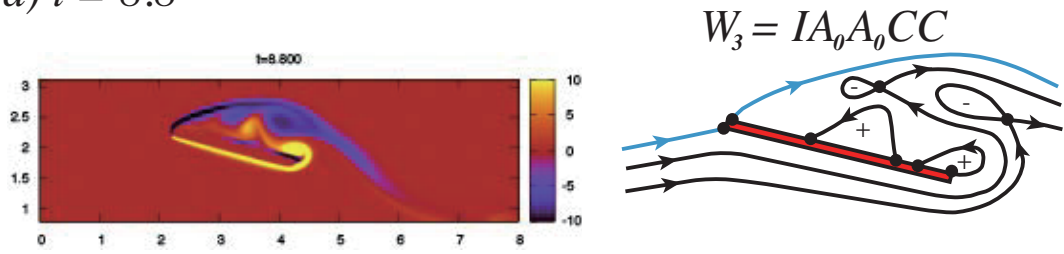

(e) $t=11.8$
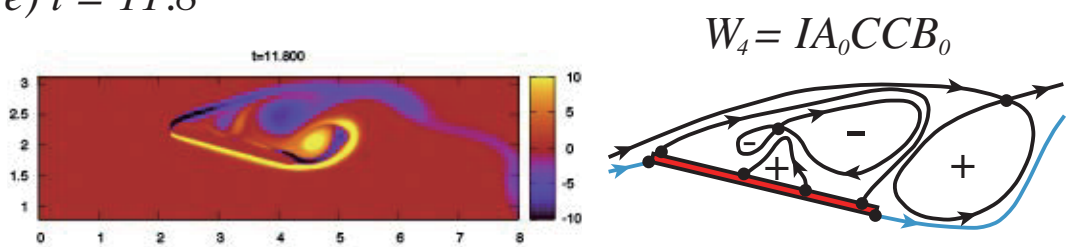

Figure 31: Evolution of the vorticity of an incompressible and viscid flow around an inclined flat plate placed in a uniform flow and the corresponding topological streamline patterns to which the unique maximal words are assigned with the algorithm given in [13]. 

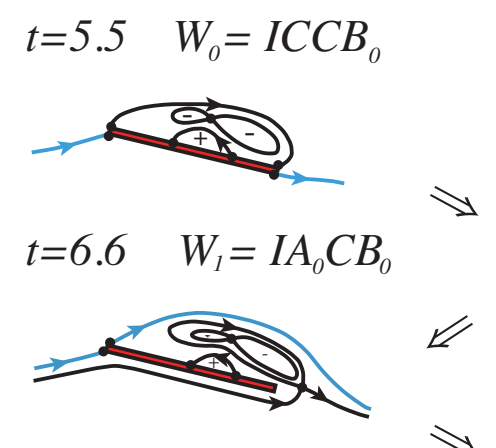

(a) $X=I M_{1, s}$
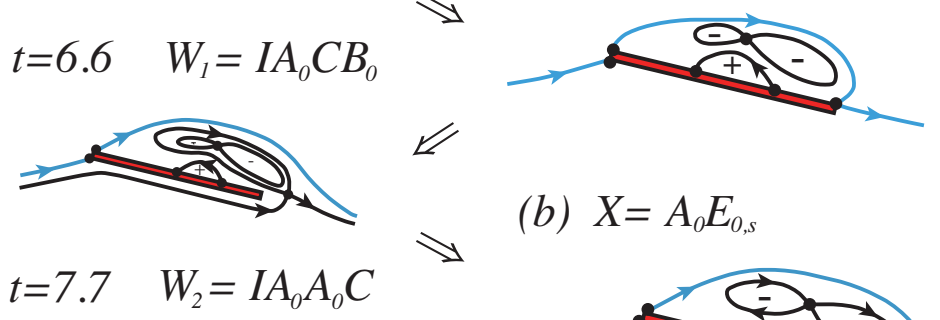

(b) $X=A_{0} E_{0, s}$
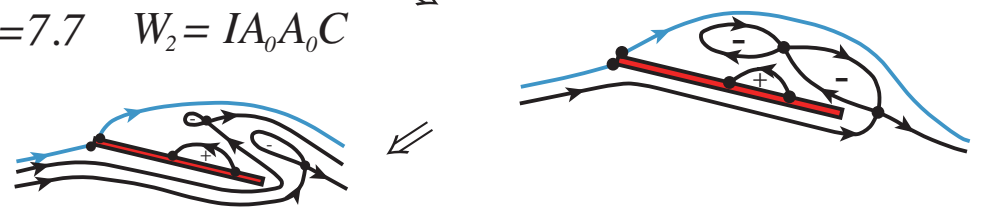

Figure 32: Transitions of the streamline topologies from $t=5.5$ to 7.7. (a) The transient pattern between the streamline patterns at $t=5.5$ and 6.6. (b) The transition between the streamline patterns at $t=6.6$ and 7.7.

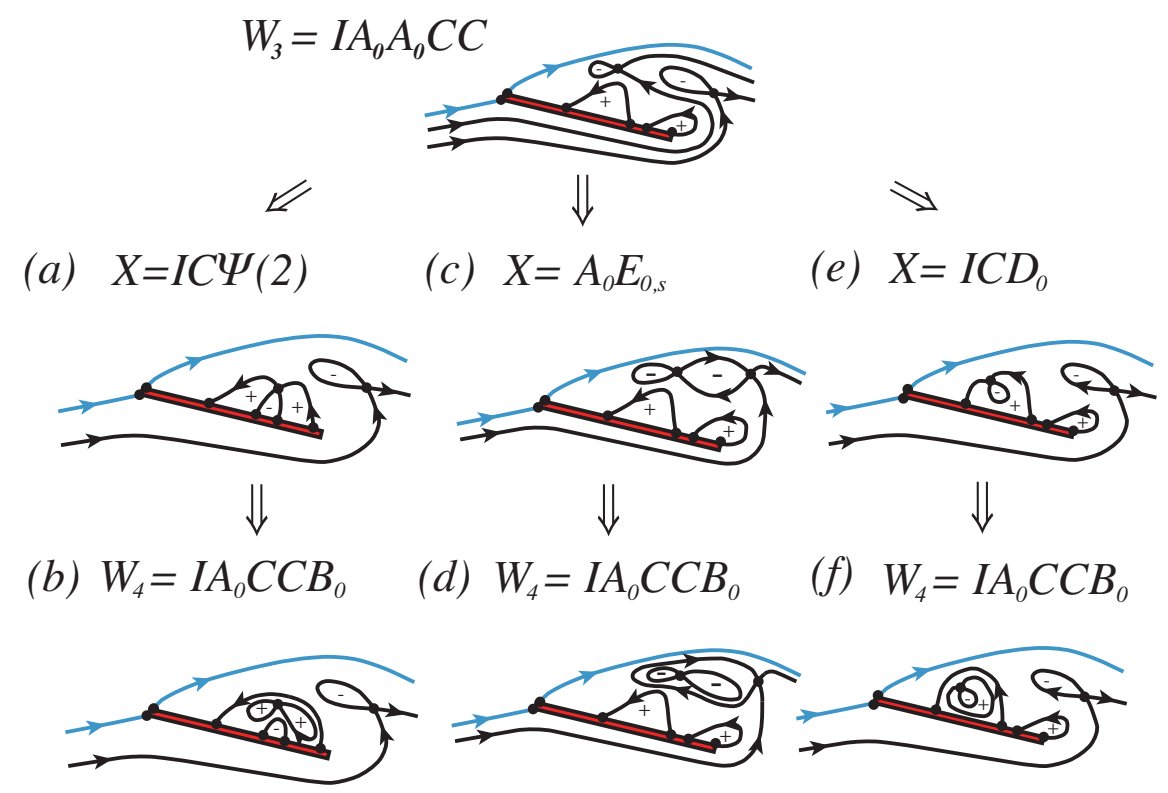

Figure 33: Three possible transitions from the streamline pattern represented by $W_{3}=I A_{0} A_{0} C C$ to that by $W_{4}=I A_{0} C C B_{0}$. 


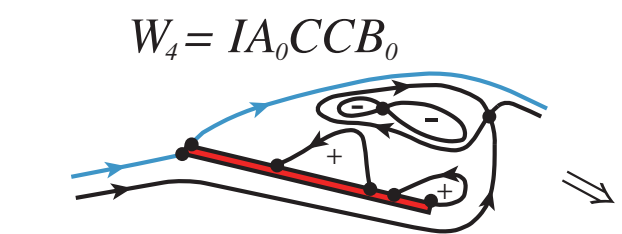

(b) $\mathrm{ICCCB}_{0}$

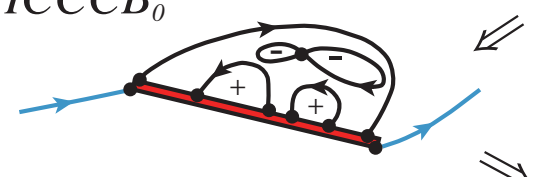

(a) $X=I M_{1, s}$

$I A_{0} C C B_{0}$

$\gg$

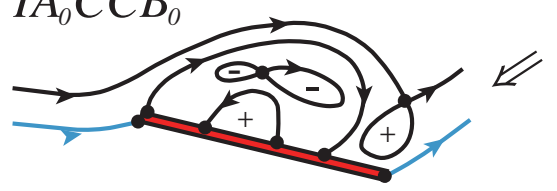

(c) $X=I C M_{1, i}$

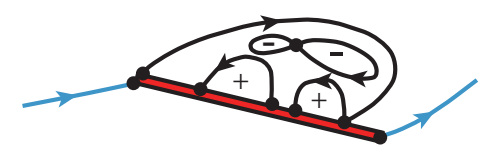

Figure 34: The shortest route of the transitions from the streamline pattern of Figure 33(d) to that of Figure 30(e) conjectured from the transition graph for the number of genus elements $M=4$. 